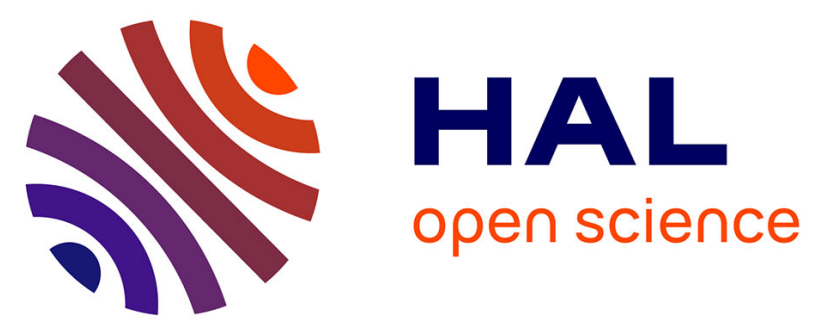

\title{
Minimization of Shear Energy in Two Dimensional Continua with Two Orthogonal Families of Inextensible Fibers: The Case of Standard Bias Extension Test
}

F. Dell'Isola, Marco Valerio d'Agostino, Angela Madeo, P. Boisse, David Steigmann

\section{To cite this version:}

F. Dell'Isola, Marco Valerio d'Agostino, Angela Madeo, P. Boisse, David Steigmann. Minimization of Shear Energy in Two Dimensional Continua with Two Orthogonal Families of Inextensible Fibers: The Case of Standard Bias Extension Test. Journal of Elasticity, 2015, 25 p. 10.1007/s10659-015-9536-3 . hal-01176313

\section{HAL Id: hal-01176313 https://hal.science/hal-01176313}

Submitted on 15 Jul 2015

HAL is a multi-disciplinary open access archive for the deposit and dissemination of scientific research documents, whether they are published or not. The documents may come from teaching and research institutions in France or abroad, or from public or private research centers.
L'archive ouverte pluridisciplinaire HAL, est destinée au dépôt et à la diffusion de documents scientifiques de niveau recherche, publiés ou non, émanant des établissements d'enseignement et de recherche français ou étrangers, des laboratoires publics ou privés. 


\title{
Minimization of Shear Energy in Two Dimensional Continua with Two Orthogonal Families of Inextensible Fibers: The Case of Standard Bias Extension Test
}

\author{
F. dell'Isola ${ }^{1,2}$ - M.V. d'Agostino ${ }^{3,4}$ - A. Madeo ${ }^{4,2}$. \\ P. Boisse B,2 D. Steigmann $^{5,2}$
}

\begin{abstract}
In this paper we consider Pipkin-type bi-dimensional continua with two orthogonal families of inextensible fibers. We generalize the representation formula due to Rivlin (J. Ration. Mech. Anal. 4(6):951-974, 1955) valid for planar placement fields. They are the sum of two vector functions each of which depends on one real variable only, are piece-wise $\mathscr{C}^{2}$ and may exhibit jumps of their first gradients on some inextensible fibers. Subsequently we consider a deformation energy depending only on the shear deformation relative to the inextensible families of fibers. In the suitably introduced space of configurations representing considered constrained kinematics, we formulate the relative energy minimization problem for the standard bias extension test problem, i.e., elongation of specimens which (i) have the shape of a rectangle with one side exactly three times longer than the other; (ii) are subject to a relative displacement of shorter sides in the direction parallel to the longer one. By exploiting the material and geometric symmetries, we reduce the aforementioned minimization problem to the determination of a piece-wise real function defined in a real interval. A delicate calculation of the energy first variation produces a necessary stationarity condition: it consists of an integral equation which is to be satisfied by the unknown function. The crucial points of this deduction are represented by (i) the reduction of two-dimensional integrals to one-dimensional integrals by the Fubini Theorem and (ii) the determination of the set of admissible kinematical functions on the basis of imposed boundary conditions, which implies a further integral constraint condition on the unknown function. Therefore the energy minimization problem requires the introduction of a global Lagrange multiplier. The established integral equations are solved numerically with a scheme based on a contraction type iterative process. Finally, the equilibrium shapes of the specimen undergoing large deformations, as determined by the presented model, are shown and briefly discussed.
\end{abstract}

\footnotetext{
$\triangle$ A. Madeo

angela.madeo@insa-1yon.fr

1 DISG, Università di Roma "La Sapienza”, Rome, Italy

2 M\&MoCS Research Center, University of L'Aquila, Cisterna di Latina, Italy

3 LaMCoS, INSA-Lyon, Villeurbanne, France

4 LGCIE, INSA-Lyon, Villeurbanne, France

5 University of California at Berkeley, Berkeley, USA
} 
Keywords Gradient elasticity · Mechanics of woven fabrics · Inextensible fibers ·

Non-local integral equations · Extensional bias test

Mathematics Subject Classification 74B20 · 74K99 · 74E99

\section{Introduction}

In their seminal papers Adkin, Rivlin [1, 26, 59, 66-68, 70, 71] and Pipkin [27, 51$57,60,61,63-65,74,77,78]$ developed a continuum model for inextensible fiber networks, to supply a predictive tool for many emerging and important technological applications. The mathematical problems which arose in their theoretical studies immediately appeared to be formidable. Indeed, they could not resolve most difficulties in a closed form and even when numerical analysis was applied to the works of Pipkin, the methods were not yet fully developed and meaningful solutions for applications were not made available. More recently investigations using these works have been continued in [17, 47, 79] motivated by aeronautical and aerospace engineering, the attention of theoretical and applied mechanicians has been attracted again to the study of mechanical systems and materials reinforced with "practically" inextensible fibers $[9,11,14,31]$. However, in many works the memory of the theoretical efforts produced by Adkin, Rivlin and Pipkin has not been emphasized and the standard first gradient Cauchy continuum model has been used in a context which had been already recognized to be unsuitable. In particular, many numerical undesired effects, such as locking (see, e.g., $[16,35,36])$ or loss of convergence, cannot be easily avoided in the presence of inextensible material lines. Moreover the associated kinematical constraints have been shown (see, e.g., [7]) to cause the onset of ill-posedness in first gradient continuum models. Indeed, the incompressibility constraint is the only kind of kinematical constraint for which one can immediately generalize the standard proof strategy developed for getting the existence and uniqueness results in the case of non-constrained first gradient continua.

In the present paper:

(i) We start by characterizing the kinematics of considered mechanical system: we limit our attention to symmetric planar systems including two families of inextensible fibers in which any admissible configuration is specifiable by means of one real function of one real variable. More details about this one-to-one characterization of the set of admissible configurations can be found in Adkin and Rivlin [1, 66, 68];

(ii) We then limit our attention to a suitable class of deformation energies $[37,38,54,56$, 65] which depend on the variation of the angle between the inextensible fibers;

(iii) We characterize the admissible equilibrium configurations of our mechanical systems as those configurations which satisfy suitable imposed displacement conditions and for which the total potential energy attains its minima;

(iv) We find that the stationary conditions associated with the principle of minimum total energy reduce to a system of integro-differential equations;

(v) We introduce an efficient iterative integration in order to numerically solve the integrodifferential equation which govern the extensional bias-test, in the particular case of a specimen having length three times larger than its width.

Actually we call standard bias test the extension test where used rectangular specimens (i) have length exactly three times longer than their width, (ii) are clamped at their shorter sides and where (iii) a relative displacement (compatible with the presence of inextensible 
Fig. 1 Space of material particles and the orthonormal system $\left(\mathscr{O}, X_{1}, X_{2}\right)$

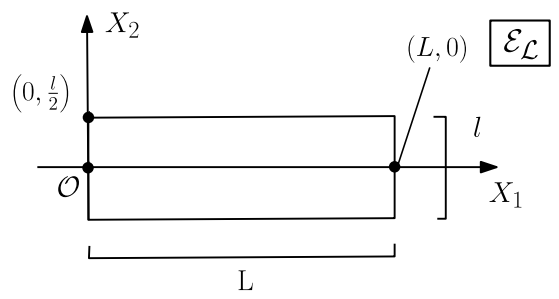

fiber) is imposed between the clamped specimen sides in the direction parallel to its longer side.

Many further investigations are motivated by the results presented herein: For instance, there is a great interest in the determination of the internal stress induced in the inextensible fibers by the imposed deformation, in order to completely describe experimental evidence (see, e.g., [41, 42]), or in the design of non-standard bias extension tests where the specimen is longer or shorter, or in designing bias tests for specimen where the inextensible fibers are not orthogonal in the reference configuration.

\section{Kinematics}

The main conceptual tool used in this paper is the principle of minimum total energy for the determination of equilibrium configurations. Therefore we must specify carefully and preliminarily the set of admissible configurations among which we look in order to find the energy minimizers.

\subsection{Geometry}

Let us consider the space of material particles of the considered continuum. It is a subset of an affine Euclidean space which we will call Lagrangian (or Material) space $\mathscr{E} \mathscr{L}$, equipped with an inner product. The material particles are placed, by a map $\mathbf{r}$, into points belonging to the current configuration included in the affine Euclidean space of positions $\mathscr{E}$ (Eulerian space). Mathematically,

$$
\mathscr{E} \mathscr{L} \supseteq B \stackrel{\mathbf{r}}{\longrightarrow} \mathbf{r}(B) \subseteq \mathscr{E} .
$$

We need to introduce a reference system on $\mathscr{E}_{\mathscr{L}}$ and, to this purpose, we choose an orthonormal system $\left(\mathscr{O}, X_{1}, X_{2}\right)$ (Fig. 1$)$.

In this paper, we assume that $B$ is a rectangle, whose sides have length $l$ and $L$, with $L=3 l$, described by the following conditions:

$$
B=\left\{\left(X_{1}, X_{2}\right) \in \mathscr{E}_{\mathscr{L}}: X_{1} \in[0, L], X_{2} \in\left[-\frac{l}{2}, \frac{l}{2}\right]\right\},
$$

and we will limit ourselves to consider the case of the standard bias extension test: Thus, we set $H=1+\frac{L}{l}=4$.

The body $B$ will be regarded as a model for a rectangular specimen of a woven fabric composed by two families of fibers which form a uniform orthogonal net intersecting the perimeter of $B$ with an angle of 45 degrees. It is convenient to introduce another orthogonal reference system, $\left(\mathscr{O}, \xi_{1}, \xi_{2}\right)$, oriented according to the directions of the fibers of the fabric 
Fig. 2 Material axes and fibers directions

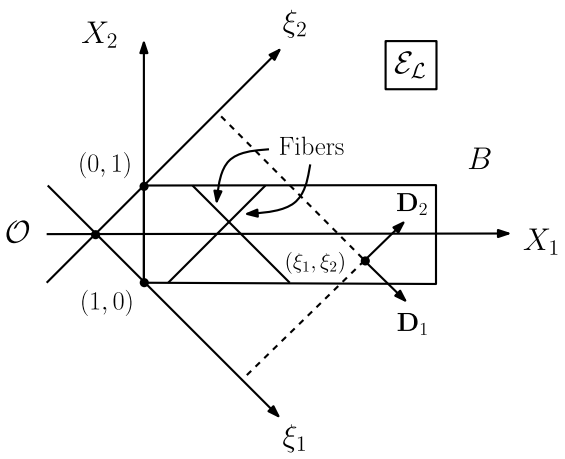

and shifted as shown in Fig. 2. In $\left(\mathscr{O}, \xi_{1}, \xi_{2}\right)$ we consider non-dimensional space coordinates, defined as follows:

$$
\xi_{1}:=\frac{1}{l}\left(X_{1}-X_{2}\right)+\frac{1}{2}, \quad \xi_{2}:=\frac{1}{l}\left(X_{1}+X_{2}\right)+\frac{1}{2} .
$$

We denote as the fiber reference the new reference system introduced and the fiber directions the two directions in which the coordinates $\xi_{1}, \xi_{2}$ are spanning. It will be useful to represent all deformation measures also in the reference system $\left(\mathscr{O}, \xi_{1}, \xi_{2}\right)$, since $\xi_{1}, \xi_{2}$ represent the orthotropic directions of the material.

We will label the generic point of $B$ by means of the coordinates $\left(\xi_{1}, \xi_{2}\right)$. We indicate with $\mathbf{D}_{1}$ and $\mathbf{D}_{2}$ the unit vectors tangent to the two families of fibers, in the tangent space $T_{\left(\xi_{1}, \xi_{2}\right)} B$ to $B$ at $\left(\xi_{1}, \xi_{2}\right)$.

We will assume that the placement field $\mathbf{r}$ :

(i) is continuous in the whole domain $B$;

(ii) has first-kind discontinuities in its first or in its second gradient at a finite number of inextensible fibers, at most;

(iii) is twice continuously differentiable in the other points of $B$.

We indicate with $\mathbf{F}$ the space gradient of $\mathbf{r}$ and we use the notation:

$$
\mathbf{d}_{1}=\mathbf{F} \cdot \mathbf{D}_{1}, \quad \mathbf{d}_{2}=\mathbf{F} \cdot \mathbf{D}_{2} .
$$

The two vectors $\mathbf{d}_{1}$ and $\mathbf{d}_{2}$ represent the directions of the fibers in the current configuration. Because of our assumptions we can say that:

(i) the inextensible fibers are not cut in the passage from the reference to the current configuration;

(ii) the current tangent vector fields of the fibers are uniquely defined for every material point except on some inextensible fibers where the tangent may suffer jumps;

(iii) the domain $B$ can be partitioned into a finite number of convex subsets in each of which the placement is of class $\mathscr{C}^{2}$.

\subsection{Inextensibility and Consequent Representation of $\mathbf{r}$}

In the proposed model, we enforce the inextensibility of the fibers of the fabric. This restricts the space of configurations because an admissible placement field must satisfy the following 
conditions:

$$
\forall\left(\xi_{1}, \xi_{2}\right) \in B, \quad\left\|\mathbf{F} \cdot \mathbf{D}_{1}\right\|^{2}=\left\|\mathbf{d}_{1}\right\|^{2}=\left\|\mathbf{F} \cdot \mathbf{D}_{2}\right\|^{2}=\left\|\mathbf{d}_{2}\right\|^{2}=1 .
$$

When $\mathbf{r} \in \mathscr{C}^{2}(\Delta)$, where $\Delta$ is an open simply connected subset of $B$, the inextensibility of the fibers implies that it is possible to decompose the placement field in the following manner (see [66]): there exist vector two fields $\mathbf{r}_{1}^{(\Delta)}\left(\xi_{1}\right)$ and $\mathbf{r}_{2}^{(\Delta)}\left(\xi_{2}\right)$, respectively defined on the projection of $\Delta$ on the fiber axes $\xi_{1}$ and $\xi_{2}$, such that

$$
\mathbf{r}_{(\Delta)}\left(\xi_{1}, \xi_{2}\right)=\mathbf{r}_{1}^{(\Delta)}\left(\xi_{1}\right)+\mathbf{r}_{2}^{(\Delta)}\left(\xi_{2}\right)
$$

We can represent these two vector fields in the basis $\left(\mathbf{D}_{1}, \mathbf{D}_{2}\right)$ as follows:

$$
\left\{\begin{array}{l}
\mathbf{r}_{1}^{(\Delta)}\left(\xi_{1}\right)=\mu_{1}^{(\Delta)}\left(\xi_{1}\right) \mathbf{D}_{1}+v_{1}^{(\Delta)}\left(\xi_{1}\right) \mathbf{D}_{2} \\
\mathbf{r}_{2}^{(\Delta)}\left(\xi_{2}\right)=v_{2}^{(\Delta)}\left(\xi_{2}\right) \mathbf{D}_{1}+\mu_{2}^{(\Delta)}\left(\xi_{2}\right) \mathbf{D}_{2},
\end{array}\right.
$$

with $\mu_{1}^{(\Delta)}, \mu_{2}^{(\Delta)}, v_{1}^{(\Delta)}, v_{2}^{(\Delta)}$, suitably regular scalar functions on the projections of $\Delta$.

Due to the conditions in (3), if we require also that $\Delta$ is convex with respect to $\xi_{1}, \xi_{2}$ segments, we can establish a specific relation between $\mu_{i}^{(\Delta)}$ and $v_{i}^{(\Delta)}, i=1,2$. If we indicate with $I_{1 \Delta}$ the projection of $\Delta$ on the axis $\xi_{1}$ and with $I_{2 \Delta}$ the projection of $\Delta$ on the axis $\xi_{2}$, we have

$$
\begin{aligned}
\left\|\mathbf{F} \cdot \mathbf{D}_{1}\right\|^{2}=1 & \Longrightarrow\left(\mu_{1,1}^{(\Delta)}\right)^{2}+\left(v_{1,1}^{(\Delta)}\right)^{2}=1 \quad \Longrightarrow \quad v_{1,1}^{(\Delta)}= \pm \sqrt{1-\left(\mu_{1,1}^{(\Delta)}\right)^{2}} \\
& \Longrightarrow v_{1}^{(\Delta)}\left(\xi_{1}\right)-v_{1}^{(\Delta)}\left(\bar{\xi}_{1}\right)= \pm \int_{\bar{\xi}_{1}}^{\xi_{1}} \sqrt{1-\left(\mu_{1,1}^{(\Delta)}(\eta)\right)^{2}} d \eta, \quad \forall \bar{\xi}_{1}, \xi_{1} \in I_{1 \Delta}, \\
\left\|\mathbf{F} \cdot \mathbf{D}_{2}\right\|^{2}=1 & \Longrightarrow\left(\mu_{2,2}^{(\Delta)}\right)^{2}+\left(v_{2,2}^{(\Delta)}\right)^{2}=1 \Longrightarrow v_{2,2}^{(\Delta)}= \pm \sqrt{1-\left(\mu_{2,2}^{(\Delta)}\right)^{2}} \\
& \Longrightarrow v_{2}^{(\Delta)}\left(\xi_{2}\right)-v_{2}^{(\Delta)}\left(\bar{\xi}_{2}\right)= \pm \int_{\bar{\xi}_{2}}^{\xi_{2}} \sqrt{1-\left(\mu_{2,2}^{(\alpha)}(\eta)\right)^{2}} d \eta, \quad \forall \bar{\xi}_{2}, \xi_{2} \in I_{2 \Delta} .
\end{aligned}
$$

This means that on any simply connected and $\left(\xi_{1}, \xi_{2}\right)$-convex subdomain $\Delta$ the whole displacement field is completely known in terms of only two real functions of one single real variable: that is, $\mu_{1}^{(\Delta)}$ and $\mu_{2}^{(\Delta)}$. In what follows, whenever square roots appear in the formulas, we will choose the sign + since only the corresponding specific families of deformations are involved in the standard bias extension test that we study herein.

Let the two lines $\Sigma_{1}$ and $\Sigma_{2}$ be defined, in the fiber reference, which are described by the following algebraic equations (see also Fig. 3):

$$
\begin{aligned}
& \Sigma_{1}:=\left\{\left(\xi_{1}, \xi_{2}\right) \in B: \xi_{1} \in[0,1], \xi_{2}=1-\xi_{1}\right\}, \\
& \Sigma_{2}:=\left\{\left(\xi_{1}, \xi_{2}\right) \in B: \xi_{1} \in[3,4], \xi_{2}=7-\xi_{1}\right\} .
\end{aligned}
$$

In this paper, we impose the following boundary conditions on the two subsets $\Sigma_{1}$ and $\Sigma_{2}$ at the boundary of $B$ :

1. vanishing displacement of the line $\Sigma_{1}$,

2. imposed displacement $\mathbf{u}_{0}=u_{0}\left(\mathbf{D}_{1}+\mathbf{D}_{2}\right)$ of the line $\Sigma_{2}$. 
Fig. 3 Discontinuity lines in the considered specimen

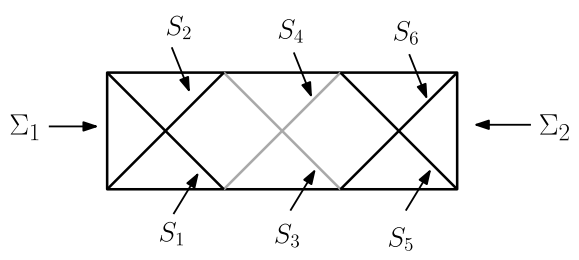

Fig. 4 Projections of the domains on the fiber axes

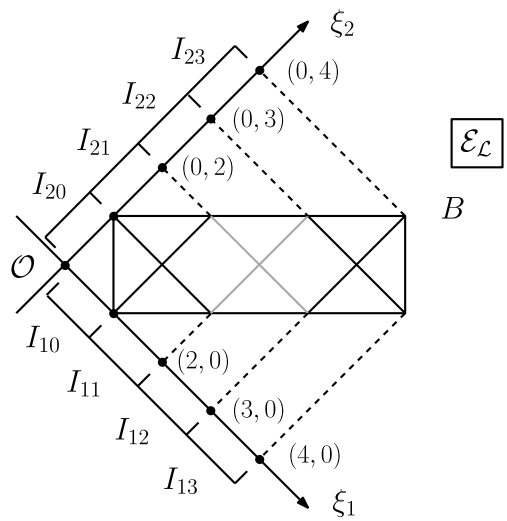

The kinematics of the bias test considered here naturally leads us to define 6 lines:

$$
\begin{array}{ll}
S_{1}:=\left\{\left(\xi_{1}, \xi_{2}\right) \in B: \xi_{1} \in[0,2], \xi_{2}=1\right\}, & S_{2}:=\left\{\left(\xi_{1}, \xi_{2}\right) \in B: \xi_{1}=1, \xi_{2} \in[0,2]\right\}, \\
S_{3}:=\left\{\left(\xi_{1}, \xi_{2}\right) \in B: \xi_{1} \in[1,3], \xi_{2}=2\right\}, & S_{4}:=\left\{\left(\xi_{1}, \xi_{2}\right) \in B: \xi_{1}=2, \xi_{2} \in[1,3]\right\}, \\
S_{5}:=\left\{\left(\xi_{1}, \xi_{2}\right) \in B: \xi_{1} \in[2,4], \xi_{2}=3\right\}, & S_{6}:=\left\{\left(\xi_{1}, \xi_{2}\right) \in B: \xi_{1}=3, \xi_{2} \in[2,4]\right\} .
\end{array}
$$

Indeed these lines are separating zones in which the inextensible fibers are subject to different kinematical conditions: As Fig. 3 shows, we remark that the part of the specimen characterized by the values of $\xi_{2} \leq 1$ (i.e., the part of the specimen below $S_{1}$ ) is constituted by all the fibers which (i) are parallel to $\xi_{1}$ and (ii) have one end blocked in $\Sigma_{1}$ while their other end is free. Exactly the same kinematical consideration is valid in the zone above $S_{5}$ (concerning all the fibers parallel to $\xi_{1}$ which have an end blocked in $\Sigma_{2}$ and the other one free) and in the zones above $S_{2}$ and under $S_{6}$ (where one has to consider the fibers parallel to $\xi_{2}$ ). The central part of the specimen is characterized kinematically as follows: The part between $S_{1}$ and $S_{3}$ is composed of fibers parallel to $\xi_{1}$ with both ends free that have interaction with fibers parallel to $\xi_{2}$ with an end fixed in $\Sigma_{1}$. Similarly, one can characterize kinematically all the subsets of the partition which we have introduced via the curves defined in Eqs. (10).

Let us denote $\pi_{1}$ and $\pi_{2}$ as the projection maps, respectively, on $\xi_{1}$ and $\xi_{2}$, and introduce the notations (see also Fig. 4)

$$
\begin{array}{ll}
I_{10}:=\pi_{1}\left(\Sigma_{1}\right)=[0,1], & I_{13}:=\pi_{1}\left(\Sigma_{2}\right)=[3,4], \\
I_{20}:=\pi_{2}\left(\Sigma_{1}\right)=[0,1], \quad I_{23}:=\pi_{2}\left(\Sigma_{2}\right)=[3,4] .
\end{array}
$$


Fig. 5 Partition of the body $B$ in different subdomains

individuated by sets of fibers with different boundary conditions or different type of interaction with the orthogonal fibers

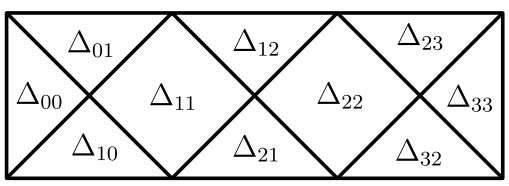

Analogously, we denote the projections of the whole $B$ on the fiber axes as (see also Fig. 4):

$$
I_{1}:=\pi_{1}(B), \quad I_{2}:=\pi_{2}(B) .
$$

In addition, considering the projections of the lines $S_{i}$ we define the following ranges:

$$
\begin{array}{rlrl}
I_{11} & :=\left[\pi_{1}\left(S_{2}\right), \pi_{1}\left(S_{4}\right)\right]=[1,2], & I_{12}:=\left[\pi_{1}\left(S_{4}\right), \pi_{1}\left(S_{6}\right)\right]=[2,3], \\
I_{21}:=\left[\pi_{2}\left(S_{1}\right), \pi_{2}\left(S_{3}\right)\right]=[1,2], & I_{23}:=\left[\pi_{2}\left(S_{3}\right), \pi_{2}\left(S_{5}\right)\right]=[2,3] .
\end{array}
$$

Then, the following partitions of the two intervals $I_{1}$ and $I_{2}$ are naturally a result on the basis of the aforementioned kinematical considerations:

$$
I_{1}=I_{10} \cup I_{11} \cup I_{12} \cup I_{13}, \quad I_{2}=I_{20} \cup I_{21} \cup I_{22} \cup I_{23} .
$$

Furthermore, the partition of the axes naturally defines a partition of $B$ into regions $\Delta_{i j}$ as follows (see Fig. 5):

$$
\Delta_{i j}:=\left(I_{1 i} \times I_{2 j}\right) \cap B, \quad B=\bigcup_{i, j=1}^{3} \Delta_{i j} .
$$

We assume that the placement field is twice continuously differentiable in any $\Delta_{i j}$. If we indicate with $\mathbf{r}^{(i, j)}\left(\xi_{1}, \xi_{2}\right)$ the restriction of $\mathbf{r}$ to the $\Delta_{i j}$ component, for the decomposition noted in (4) we have

$$
\mathbf{r}^{(i, j)}\left(\xi_{1}, \xi_{2}\right)=\mathbf{r}_{1}^{(i)}\left(\xi_{1}\right)+\mathbf{r}_{2}^{(j)}\left(\xi_{2}\right)=:\left(\mu_{1}^{(i)}\left(\xi_{1}\right)+v_{2}^{(j)}\left(\xi_{2}\right)\right) \mathbf{D}_{1}+\left(v_{1}^{(i)}\left(\xi_{1}\right)+\mu_{2}^{(j)}\left(\xi_{2}\right)\right) \mathbf{D}_{2},
$$

as the global continuity condition of $\mathbf{r}$ allows us to identify the vector fields $\mathbf{r}_{1}^{(i)}\left(\xi_{1}\right), \mathbf{r}_{2}^{(j)}\left(\xi_{2}\right)$ along straight lines included in $B$.

Indeed, if we consider two regions $\Delta_{i j}$ and $\Delta_{i k}$ then we have that $\mathbf{r}_{1}^{\left(\Delta_{i j}\right)}\left(\xi_{1}\right)=\mathbf{r}_{1}^{\Delta_{i k}}\left(\xi_{1}\right)=$ : $\mathbf{r}_{1}^{(i)}\left(\xi_{1}\right)$ and analogously, when considering two regions $\Delta_{i j}$ and $\Delta_{h j}$, one has $\mathbf{r}_{2}^{\left(\Delta_{i j}\right)}\left(\xi_{2}\right)=$ $\mathbf{r}_{2}^{\Delta_{k j}}\left(\xi_{2}\right)=: \mathbf{r}_{2}^{(j)}\left(\xi_{2}\right)$.

\subsection{Space of Configurations for Considered System}

A number of properties and results can be proven for the scalar fields which determine the displacement field. The regularity assumptions, which we have posed together with the condition of inextensibility of the fibers and the boundary conditions, will indeed determine the space of configurations inside of which we seek the equilibrium configurations: i.e., those configurations which minimize the total energy.

\subsubsection{Restrictions on the Fields $\mu$ and $v$ Imposed by Boundary Conditions}

We prove here the three following properties of the displacement field for the bias test problem with inextensible fibers: 
1. the boundary condition on $\Sigma_{1}$ implies that $\mathbf{r}\left(\xi_{1}, \xi_{2}\right)=\xi_{1} \mathbf{D}_{1}+\xi_{2} \mathbf{D}_{2} \forall\left(\xi_{1}, \xi_{2}\right) \in \Delta_{00}$,

2. the boundary conditions on $\Sigma_{2}$ implies that $\mathbf{r}\left(\xi_{1}, \xi_{2}\right)=\xi_{1} \mathbf{D}_{1}+\xi_{2} \mathbf{D}_{2}+\mathbf{u}_{0} \forall\left(\xi_{1}, \xi_{2}\right) \in \Delta_{33}$,

3 . the continuity of $\mathbf{r}$ at the point $(3,3)$ yields a system (which is specified at the end of this section) of integral conditions for determining the two functions $\mu_{1}\left(\xi_{1}\right)$ and $\mu_{2}\left(\xi_{2}\right)$.

Proposition 1 Let $\mathbf{r}^{(0,0)}$ be the restriction of $\mathbf{r}$ to the subdomain $\Delta_{00}$. If the displacement vanishes on $\Sigma_{1}$, then we have $\mathbf{r}^{(0,0)}\left(\xi_{1}, \xi_{2}\right)=\xi_{1} \mathbf{D}_{1}+\xi_{2} \mathbf{D}_{2} \forall\left(\xi_{1}, \xi_{2}\right) \in \Delta_{00}$.

Proof We consider the following parametric description of $\Sigma_{1}$ :

$$
\Sigma_{1}=\left\{\left(\xi_{1}(t), \xi_{2}(t)\right): \xi_{1}(t)=t, \xi_{2}(t)=1-t \text { with } t \in[0,1]\right\}
$$

and we note that

$$
\begin{aligned}
\mathbf{r}^{(0,0)}\left(\xi_{1}(t), \xi_{2}(t)\right) & =\mathbf{r}_{1}^{(0)}\left(\xi_{1}(t)\right)+\mathbf{r}_{2}^{(0)}\left(\xi_{2}(t)\right) \\
& =\left(\mu_{1}^{(0)}\left(\xi_{1}(t)\right)+v_{2}^{(0)}\left(\xi_{2}(t)\right)\right) \mathbf{D}_{1}+\left(v_{1}^{(0)}\left(\xi_{1}(t)\right)+\mu_{2}^{(0)}\left(\xi_{2}(t)\right)\right) \mathbf{D}_{2},
\end{aligned}
$$

which, because of the imposed boundary conditions, imply

$$
\left\{\begin{array}{l}
\mu_{1}^{(0)}\left(\xi_{1}(t)\right)+v_{2}^{(0)}\left(\xi_{2}(t)\right)=t \\
v_{1}^{(0)}\left(\xi_{1}(t)\right)+\mu_{2}^{(0)}\left(\xi_{2}(t)\right)=1-t .
\end{array}\right.
$$

By differentiating the two equations in (21) with respect to $t$ we obtain:

$$
\left\{\begin{array} { l } 
{ \frac { d \mu _ { 1 } ^ { ( 0 ) } } { d \xi _ { 1 } } | _ { t } - \frac { d \nu _ { 2 } ^ { ( 0 ) } } { d \xi _ { 2 } } | _ { 1 - t } = 1 } \\
{ \frac { d v _ { 1 } ^ { ( 0 ) } } { d \xi _ { 1 } } | _ { t } - \frac { d \mu _ { 2 } ^ { ( 0 ) } } { d \xi _ { 2 } } | _ { 1 - t } = - 1 }
\end{array} \Longrightarrow \left\{\begin{array}{l}
\mu_{1,1}^{(0)}=1+v_{2,2}^{(0)} \\
v_{1,1}^{(0)}=\mu_{2,2}^{(0)}-1
\end{array}\right.\right.
$$

Recalling the relation $1=\left(\mu_{1,1}^{(0)}\right)^{2}+\left(v_{1,1}^{(0)}\right)^{2}$, we obtain

$$
\begin{aligned}
1 & =\left(\mu_{1,1}^{(0)}\right)^{2}+\left(v_{1,1}^{(0)}\right)^{2}=\left(1+v_{2,2}^{(0)}\right)^{2}+\left(\mu_{2,2}^{(0)}-1\right)^{2} \\
& =\underbrace{\left(\mu_{2,2}^{(0)}\right)^{2}+\left(v_{2,2}^{(0)}\right)^{2}}_{=1}+2-2 \mu_{2,2}^{(0)}+2 v_{2,2}^{(0)}=3+2\left(v_{2,2}^{(0)}-\mu_{2,2}^{(0)}\right) .
\end{aligned}
$$

As a consequence

$$
1=3+2\left(v_{2,2}^{(0)}-\mu_{2,2}^{(0)}\right) \quad \Longrightarrow \quad \mu_{2,2}^{(0)}-v_{2,2}^{(0)}=1 .
$$

Therefore we can easily find the fields $\mu_{2,2}^{(0)}$ and $\nu_{2,2}^{(0)}$. Indeed,

$$
\left\{\begin{array}{l}
\left(\mu_{2,2}^{(0)}\right)^{2}+\left(v_{2,2}^{(0)}\right)^{2}=1 \\
\mu_{2,2}^{(0)}-v_{2,2}^{(0)}=1
\end{array} \quad \Longrightarrow \quad v_{2,2}^{(0)}=0 \vee v_{2,2}^{(0)}=-1\right.
$$


but, restricting to the case $v_{2,2}^{(0)} \in[0, \sqrt{2} / 2]$, we need consider only the solution $v_{2,2}^{(0)}=0$. As a consequence $\mu_{2,2}^{(0)}=1$.

In the same way we find that $v_{1,1}^{(0)}=0$ and $\mu_{1,1}^{(0)}=1$ for any $\xi_{1} \in I_{10}$.

Integrating the corresponding expressions we have

$$
\begin{cases}\mu_{1}^{(0)}\left(\xi_{1}\right)=\xi_{1}+\mu_{1}^{(0)}(0) & \xi_{1} \in I_{10} \\ v_{1}^{(0)}\left(\xi_{1}\right)=v_{1}^{(0)}(0) & \xi_{1} \in I_{10} \\ \mu_{2}^{(0)}\left(\xi_{2}\right)=\xi_{2}+\mu_{2}^{(0)}(0) & \xi_{2} \in I_{20} \\ v_{2}^{(0)}\left(\xi_{2}\right)=v_{2}^{(0)}(0) & \xi_{2} \in I_{20} .\end{cases}
$$

In the decomposition (18) the functions $\mathbf{r}_{1}$ and $\mathbf{r}_{2}$ are determined up to an additive vector constant (i.e., up to two scalar constants). We can choose the constant to satisfy:

$$
\mu_{1}^{(0)}(0)=v_{1}^{(0)}(0)=\mu_{2}^{(0)}(0)=v_{2}^{(0)}(0)=0
$$

In a similar way, ${ }^{1}$ we can prove also the following

Proposition 2 Let $\mathbf{r}^{(3,3)}$ be the restriction of $\mathbf{r}$ to the subdomain $\Delta_{33}$ and $\mathbf{u}_{0}$ the imposed displacement on $\Sigma_{2}$. Then we have $\mathbf{r}^{(3,3)}\left(\xi_{1}, \xi_{2}\right)=\xi_{1} \mathbf{D}_{1}+\xi_{2} \mathbf{D}_{2}+\mathbf{u}_{0}$.

Remark 1 It is very important to remark that in every region $\Delta_{i j}$ the two vector fields $\mathbf{r}_{1}^{(i)}\left(\xi_{1}\right)$ and $\mathbf{r}_{2}^{(j)}\left(\xi_{2}\right)$ in the decomposition

$$
\mathbf{r}^{(i, j)}\left(\xi_{1}, \xi_{2}\right)=\mathbf{r}_{1}^{(i)}\left(\xi_{1}\right)+\mathbf{r}_{2}^{(j)}\left(\xi_{2}\right),
$$

are defined up to two additive constants.

Indeed, if we take

$$
\mathbf{r}_{1}^{(i)}\left(\xi_{1}\right)=\mu_{1}^{(i)}\left(\xi_{1}\right) \mathbf{D}_{1}+v_{1}^{(i)}\left(\xi_{1}\right) \mathbf{D}_{2}, \quad \mathbf{r}_{2}^{(j)}\left(\xi_{2}\right)=v_{2}^{(j)}\left(\xi_{2}\right) \mathbf{D}_{1}+\mu_{2}^{(j)}\left(\xi_{2}\right) \mathbf{D}_{2},
$$

then it is immediately evident that also for

$$
\begin{aligned}
& \overline{\mathbf{r}}_{1}^{(i)}\left(\xi_{1}\right)=\left(\mu_{1}^{(i)}\left(\xi_{1}\right)+\alpha_{i}\right) \mathbf{D}_{1}+\left(v_{1}^{(i)}\left(\xi_{1}\right)+\beta_{j}\right) \mathbf{D}_{2}, \\
& \overline{\mathbf{r}}_{2}^{(j)}\left(\xi_{2}\right)=\left(v_{2}^{(j)}\left(\xi_{2}\right)-\alpha_{i}\right) \mathbf{D}_{1}+\left(\mu_{2}^{(j)}\left(\xi_{2}\right)-\beta_{j}\right) \mathbf{D}_{2}
\end{aligned}
$$

with $\alpha_{i}, \beta_{j} \in \mathbb{R}$, we have

$$
\mathbf{r}^{(i, j)}\left(\xi_{1}, \xi_{2}\right)=\overline{\mathbf{r}}_{1}^{(i)}\left(\xi_{1}\right)+\overline{\mathbf{r}}_{2}^{(j)}\left(\xi_{2}\right)=\mathbf{r}_{1}^{(i)}\left(\xi_{1}\right)+\mathbf{r}_{2}^{(j)}\left(\xi_{2}\right) .
$$

Now, we can define the four functions $\mu_{1}, v_{1}, \mu_{2}$ and $v_{2}$ in the intervals $I_{1}$ and $I_{2}$ respectively as follows:

$$
\begin{aligned}
& \left(\forall \xi_{1} \in I_{1 j}\right) \quad\left(\mu_{1}\left(\xi_{1}\right)=\mu_{1}^{(j)}\left(\xi_{1}\right), v_{1}\left(\xi_{1}\right)=v_{1}^{(j)}\left(\xi_{1}\right)\right), \\
& \left(\forall \xi_{2} \in I_{2 j}\right) \quad\left(\mu_{2}\left(\xi_{2}\right)=\mu_{2}^{(j)}\left(\xi_{2}\right), v_{2}\left(\xi_{2}\right)=v_{2}^{(j)}\left(\xi_{2}\right)\right) .
\end{aligned}
$$

${ }^{1}$ One could also apply the previous proposition to the function $\mathbf{r}^{(3,3)}\left(\xi_{1}, \xi_{2}\right)-\mathbf{u}_{0}$. 
Obviously it is easy to see that we can determine (uniquely) the constants left arbitrary by the decomposition formula for displacement, after the initial choice in (28), simply by demanding the continuity on the intervals $I_{1}$ and $I_{2}$ of the four functions just introduced.

\subsubsection{Continuity Conditions in $(3,3)$}

Because of (18), (6), (7), (33) and (34), the continuity conditions of the placement field $\mathbf{r}$ in $(3,3)$ with respect to the functions $\mu_{1}, \mu_{2}$ can be written as:

$$
\left\{\begin{array}{l}
\mu_{1}(3)+\int_{1}^{3} \sqrt{1-\left(\mu_{2,2}(\eta)\right)^{2}} d \eta=3+u_{0} \\
\mu_{2}(3)+\int_{1}^{3} \sqrt{1-\left(\mu_{1,1}(\eta)\right)^{2}} d \eta=3+u_{0} .
\end{array}\right.
$$

This condition is a consequence of the continuity of displacement in $B$ and of Proposition 2, which implies that the subbody $\Delta_{33}$ is simply rigidly translating.

\subsection{Symmetry Conditions}

In this section we characterize the displacement fields which are symmetric with respect to the $X_{1}$-axis. This means that, given a point $P$ of coordinates $(\xi, \eta)$ and its symmetric point $P_{s}$ having coordinates $(\eta, \xi)$, the following conditions hold:

$$
\left\{\begin{array} { l } 
{ \mathbf { d } _ { 1 } ( P ) \cdot \mathbf { D } _ { 1 } = \mathbf { d } _ { 2 } ( P _ { s } ) \cdot \mathbf { D } _ { 2 } } \\
{ \mathbf { d } _ { 1 } ( P ) \cdot \mathbf { D } _ { 2 } = \mathbf { d } _ { 2 } ( P _ { s } ) \cdot \mathbf { D } _ { 1 } }
\end{array} \Longrightarrow \left\{\begin{array}{l}
\mu_{1,1}(\xi)=\mu_{2,2}(\xi) \\
v_{1,1}(\eta)=v_{2,2}(\eta) .
\end{array}\right.\right.
$$

Considering the symmetry of boundary conditions and the first equality in (36), we get directly the following identity:

$$
\mu_{1}(\xi)=\mu_{2}(\xi)=: \mu(\xi) \quad \forall \xi \in[0,4] .
$$

Therefore, considering the relations between $\mu_{1}, \mu_{2}$ and $v_{1}, v_{2}$, the kinematics of the symmetric bias extension problem is completely described by means of a unique field $\mu$. It is possible to determine another symmetry condition for the placement field. Indeed, our problem is equivalent (up to a translation) to the one which is obtained by imposing the following boundary conditions:

1. imposed displacement $-\mathbf{u}_{0} / 2=-u_{0} / 2\left(\mathbf{D}_{1}+\mathbf{D}_{2}\right)$ of the line $\Sigma_{1}$,

2. imposed displacement $\mathbf{u}_{0} / 2=u_{0} / 2\left(\mathbf{D}_{1}+\mathbf{D}_{2}\right)$ of the line $\Sigma_{2}$.

This implies the symmetry of $\mathbf{r}$ with respect to the line parallel to the $X_{2}$-axis passing through the point with coordinates $(2,2)$ in the $\xi_{1} \xi_{2}$-reference. It is straightforward to deduce that the functions $\mu_{1,1}$ and $\mu_{2,2}$ are even with respect to the points $\xi_{1}=2$ and $\xi_{2}=2$ in their domains of definition. Since the derivative of a even function is always an odd function, and in general the derivative of $\mu_{1,1}$ and $\mu_{2,2}$ is not equal to zero at $\xi_{1}=2$ and $\xi_{2}=2$, the second derivative of the fields $\mu_{1}$ and $\mu_{2}$ can be discontinuous in these points.

\subsection{First Variations of the Fields Belonging to the Space of Configurations}

If we assume the hypothesis of symmetry, the configuration of body $B$ is characterized by only one scalar field $\mu(\xi)$, defined on the real interval $[0,4]$. However, having already determined $\mu$ on $[0,1]$ and $[3,4]$ by means of imposed boundary conditions, we are left to 
find $\mu$ on $I=[1,3]$. Due to Proposition 1 , the continuity of the function $\mu$ and (35), we have also the conditions

$$
\mu(1)=1, \quad \mu(3)+\int_{1}^{3} \sqrt{1-(\mu, \xi)^{2}} d \eta=3+u_{0},
$$

which must be satisfied by the functions in the space of configurations. Therefore the space of configurations is constituted by the set of functions $\mu$ in $\mathscr{C}_{p w}^{2}(I,\{2\})$, that is, the space of two times continuously differentiable functions on $I$ whose second derivatives can jump at 2, which satisfy the conditions (38).

Therefore, in order to be kinematically admissible, a variation $\delta \mu$ of $\mu$ has to meet the following conditions:

$$
\delta \mu(1)=0, \quad \mu(3)+\delta \mu(3)+\int_{1}^{3} \sqrt{1-\left(\mu_{, \xi}+\delta \mu_{, \xi}\right)^{2}} d \eta=u_{0}+3 .
$$

First, we subtract the integral condition (38) from (39) to obtain:

$$
\delta \mu(3)+\int_{1}^{3} \sqrt{1-\left(\mu_{, \xi}+\delta \mu_{, \xi}\right)^{2}} d \eta-\int_{1}^{3} \sqrt{1-\left(\mu_{, \xi}\right)^{2}} d \eta=0 .
$$

Developing the argument of the first square root to the first order, ${ }^{2}$ we obtain

$$
\delta \mu(3)+\int_{1}^{3}\left(\sqrt{1-(\mu, \xi)^{2}}-\frac{\mu^{\prime}}{\sqrt{1-\left(\mu_{, \xi}\right)^{2}}} \delta \mu, \xi\right) d \eta-\int_{1}^{3} \sqrt{1-\left(\mu_{, \xi}\right)^{2}} d \eta=0
$$

and, consequently,

$$
\delta \mu(3)-\int_{1}^{3} \frac{\mu_{, \xi}}{\sqrt{1-\left(\mu_{, \xi}\right)^{2}}} \delta \mu_{, \xi} d \eta=0 .
$$

Noting that we can write $\delta \mu(3)$ as $\int_{1}^{3} \delta \mu_{, \xi} d \eta$, we find that (42) is equivalent to

$$
\int_{1}^{3}\left(1-\frac{\mu_{, \xi}}{\sqrt{1-\left(\mu_{, \xi}\right)^{2}}}\right) \delta \mu_{, \xi} d \eta=0 .
$$

This last orthogonality condition characterizes the admissible first variations of the functions in the space of configurations.

\section{First Variation of Energy}

We assume the following expression for the energy density:

$$
W\left(\xi_{1}, \xi_{2}\right)=\frac{1}{2}\left(\mathbf{d}_{1} \cdot \mathbf{d}_{2}\right)^{2},
$$

which, once expressed in terms of the kinematical field $\mu$, becomes

$$
W\left(\xi_{1}, \xi_{2}\right)=\frac{1}{2}\left(\left(\mu_{, \xi}\left(\xi_{1}\right)\right)^{2}\left(1-\left(\mu_{, \xi}\left(\xi_{2}\right)\right)^{2}\right)+\left(\mu_{, \xi}\left(\xi_{2}\right)\right)^{2}\left(1-\left(\mu_{, \xi}\left(\xi_{1}\right)\right)^{2}\right)\right.
$$

\footnotetext{
${ }^{2}$ Note that this development is not possible in the neighborhood of the reference configuration.
} 


$$
+2 \mu_{, \xi}\left(\xi_{1}\right) \mu_{, \xi}\left(\xi_{2}\right) \sqrt{\left.\left(1-\left(\mu_{, \xi}\left(\xi_{1}\right)\right)^{2}\right)\left(1-\left(\mu_{, \xi}\left(\xi_{2}\right)\right)^{2}\right)\right)} .
$$

In order to minimize the energy in the space of configurations, we have to introduce a Lagrange multiplier to take into account the integral constraint (38) imposed on $\mu$. By using the notation

$$
C(\mu, \xi):=\mu(3)+\int_{1}^{3} \sqrt{1-\left(\mu_{, \xi}\right)^{2}} d \eta-3-u_{0},
$$

we then consider the following energy functional:

$$
\mathscr{A}_{W}=\int_{B} W d m+\Lambda C(\mu, \xi), \quad \text { with } \Lambda \in \mathbb{R} .
$$

Its first variation is

$$
\delta \mathscr{A}_{W}=\int_{B} \delta W d m+\Lambda \delta C\left(\mu_{, \xi}\right)+C\left(\mu_{, \xi}\right) \delta \Lambda .
$$

In the Appendix we show all the details of the calculations for obtaining the aforementioned variation: to present them in the most effective way it is useful to introduce four integral operators $\mathscr{A}_{i}$ and $\mathscr{D}_{i}$, where $i=1,2$. These operators are assumed to map a function $f$ defined in the interval $[0,4]$ into a function defined respectively in $J_{1}=[1,2], J_{2}=[2,3]$ and are defined by means of the following equalities:

$$
\begin{aligned}
& \left\{\begin{array}{l}
{\left[\mathscr{A}_{1}(f)\right](\xi)=\int_{1}^{\xi+1} f(\eta) \sqrt{1-(f(\eta))^{2}} d \eta} \\
{\left[\mathscr{D}_{1}(f)\right](\xi)=\left(1-\int_{1}^{\xi+1}\left(1-(f(\eta))^{2}\right) d \eta\right),}
\end{array}\right. \\
& \left\{\begin{array}{l}
{\left[\mathscr{A}_{2}(f)\right](\xi)=\int_{\xi-1}^{3} f(\eta) \sqrt{1-(f(\eta))^{2}} d \eta} \\
{\left[\mathscr{D}_{2}(f)\right](\xi)=\left(1-\int_{\xi-1}^{3}\left(1-(f(\eta))^{2}\right) d \eta\right) .}
\end{array}\right.
\end{aligned}
$$

Remark It is easy to check that for every function $f$ if $\sup |f| \leq 1$ then $\sup \left|\mathscr{A}_{i}(f)\right| \leq 1$ and $\sup \left|\mathscr{D}_{i}(f)\right| \leq 1$, where $i=1,2$ and the sup is estimated in the corresponding domain of definition.

The stationary condition finally obtained is expressed by the following integro-differential equations (to be complemented by suitable boundary conditions):

$$
\frac{1-2\left(\mu_{, \xi}\right)^{2}}{2 \sqrt{1-\left(\mu_{, \xi}\right)^{2}}} \mathscr{A}_{i}\left(\mu_{, \xi}\right)-\mu_{, \xi} \mathscr{D}_{i}\left(\mu_{, \xi}\right)+\Lambda\left(1-\frac{\mu_{, \xi}}{\sqrt{1-\left(\mu_{, \xi}\right)^{2}}}\right)=0 \quad \forall \xi \in J_{i}, i=1,2,
$$

which can be transformed to the form

$$
\left(\frac{1-2\left(\mu_{, \xi}\right)^{2}}{2 \sqrt{1-\left(\mu_{, \xi}\right)^{2}}} \mathscr{A}_{i}\left(\mu_{, \xi}\right)-\mu_{, \xi} \mathscr{D}_{i}\left(\mu_{, \xi}\right)\right)\left(\frac{\mu_{, \xi}}{\sqrt{1-\left(\mu_{, \xi}\right)^{2}}}-1\right)^{-1}=\Lambda \quad \forall \xi \in J_{i}, i=1,2
$$


To proceed in the study of this integro-differential equation, it is useful to introduce an auxiliary function $F$ defined on the domain

$$
\mathbb{R}^{3} \supseteq D:=\left[\frac{\sqrt{2}}{2}, 1\right) \times(0,1] \times(0,1]
$$

as follows:

$$
F(x, a, d)=\left(\frac{1-2 x^{2}}{2 \sqrt{1-x^{2}}} a-x d\right)\left(\frac{x}{\sqrt{1-x^{2}}}-1\right)^{-1} .
$$

It can be checked that $F(D)=\mathbb{R}^{-}$and that there exists a function

$$
G:(0,1] \times(0,1] \times \mathbb{R}^{-} \rightarrow\left[\frac{\sqrt{2}}{2}, 1\right)
$$

such that

$$
F(G(a, d, \Lambda), a, d)=\Lambda ; \quad G(a, d, F(x, a, d))=x .
$$

By means of the functions $F$ and $G$, the stationary problem can be formulated in the two following more compact forms.

Problem 4 Find a function $\hat{\mu} \in \mathscr{C}_{p w}^{2}(I,\{2\})$ such that i) $\hat{\mu}(1)=1$, and ii) there exists a negative real number $\Lambda$ such that the following equations are satisfied:

$$
\left\{\begin{array}{l}
F\left(\hat{\mu}_{, \xi}, \mathscr{A}_{i}\left(\hat{\mu}_{, \xi}\right), \mathscr{D}_{i}\left(\hat{\mu}_{, \xi}\right)\right)=\Lambda \quad \forall \xi \in J_{i}, i=1,2 \\
C(\hat{\mu}, \xi)=0 .
\end{array}\right.
$$

By using the second of identities (53) and conditions (54) it can be easily seen that Problem 4 is equivalent to the following one, which is more suitable to the application of the Picard-type iteration method we will use in the following section:

Problem 5 Find a function $\mu_{*} \in \mathscr{C}_{p w}^{2}(I,\{2\})$ such that

$$
\left\{\begin{array}{l}
\mu_{*}(\xi)=1+\int_{1}^{\xi} G\left(\left[\mathscr{A}_{1}\left(\mu_{*, \xi}\right)\right](\eta),\left[\mathscr{D}_{1}\left(\mu_{*, \xi}\right)\right](\eta), \Lambda\right) d \eta \quad \forall \xi \in[1,2] \\
\left.\left.\mu_{*}(\xi)=\mu_{*}(2)+\int_{2}^{\xi} G\left(\left[\mathscr{A}_{2}\left(\mu_{*, \xi}\right)\right](\eta),\left[\mathscr{D}_{2}\left(\mu_{*, \xi}\right)\right](\eta), \Lambda\right) d \eta \quad \forall \xi \in\right] 2,3\right],
\end{array}\right.
$$

choosing the parameter $\Lambda$ in order to meet the integral condition $C\left(\mu_{*, \xi}\right)=0$.

Postponing the study of the well-posedness of the formulated integro-differential problem to further investigations we show that the last presented form more easily lends itself to numerical integration.

\section{Numerical Resolution of the Problem}

The problem expressed by (54) cannot generally be solved in closed form: therefore we are obliged to resort to numerical methods. 


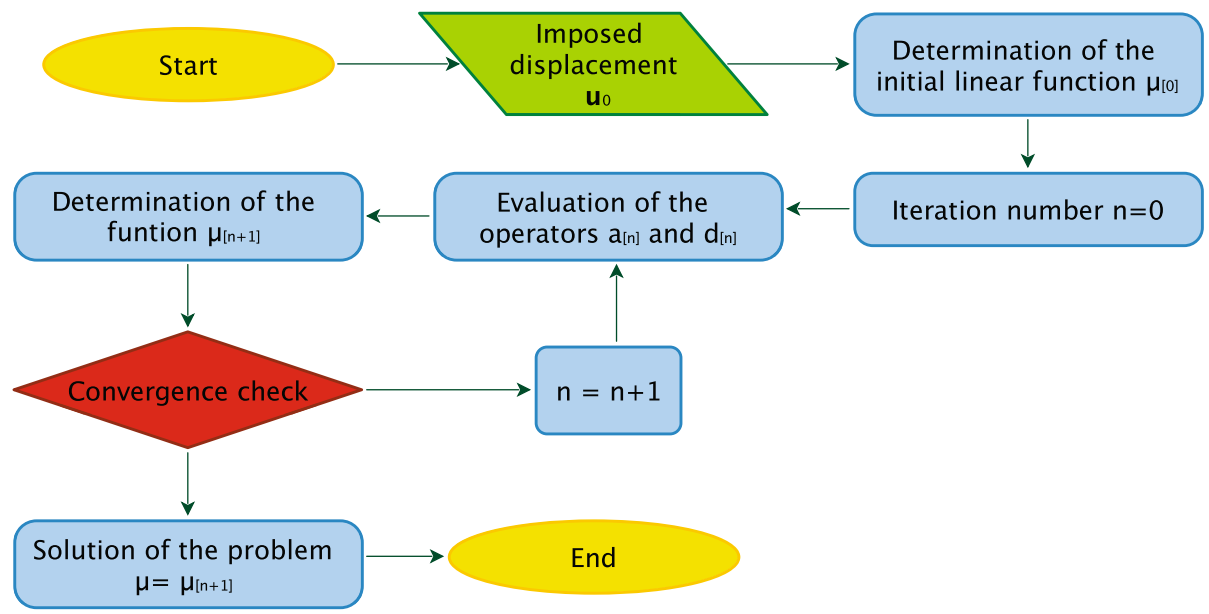

Fig. 6 Resolutive algorithm

In order to solve the problem (55) by means of numerical integration techniques we define an iterative scheme in which the initial function $\mu_{[0]}$ is chosen to be a first order polynomial satisfying the boundary conditions (38).

Given the approximation of the solution of the Picard-type problem formulated in (55) at the step $n-1$, that is, the function $\mu_{[n-1]}$, we find the $n$-th approximation as follows:

$$
\left\{\begin{array}{l}
\mu_{[n]}(\xi)=1+\int_{1}^{\xi} G\left(\left[\mathscr{A}_{1}\left(\mu_{[n-1], \xi}\right)\right](\eta),\left[\mathscr{D}_{1}\left(\mu_{[n-1], \xi}\right)\right](\eta), \Lambda_{n}\right) d \eta \quad \forall \xi \in[1,2] \\
\left.\left.\mu_{[n]}(\xi)=\mu_{[n]}(2)+\int_{2}^{\xi} G\left(\left[\mathscr{A}_{2}\left(\mu_{[n-1], \xi}\right)\right](\eta),\left[\mathscr{D}_{2}\left(\mu_{[n-1], \xi}\right)\right](\eta), \Lambda_{n}\right) d \eta \quad \forall \xi \in\right] 2,3\right],
\end{array}\right.
$$

choosing the parameter $\Lambda_{n}$ in order to meet the boundary condition $C\left(\mu_{[n], \xi}\right)=0$.

We iterate until the numerical convergence of the solution is obtained. In Fig. 6 the flow chart of the algorithm is shown.

\subsection{Numerical Results}

The algorithm is implemented in Mathematica ${ }^{\circledR}$ with different values of the imposed displacement $u_{0}$. In Fig. 7 we show the deformed geometry for 3 different imposed displacements. The results match qualitatively the experimental results obtained with the standard bias extension text for composite fiber reinforcements.

The numerical analysis shows an interesting phenomenon which deserves some discussion and further theoretical, numerical and experimental investigations.

Indeed in the numerical solutions obtained, in general, one can check that the scalar field giving the angle between the inextensible fibers (fiber shear angle), easily related to the field $\mathbf{d}_{1} \cdot \mathbf{d}_{2}$, is not piece-wise constant in the kinematically determined (see previous sections) different zones of the domain as it is usually stated in the literature on fiber composite reinforcements. Actually the plots of this field along lines parallel to the long side of the specimen also show different concavities for different imposed displacements and it is also possible to observe the existence of a unique critical displacement for which the fiber shear angle is piece-wise constant. 

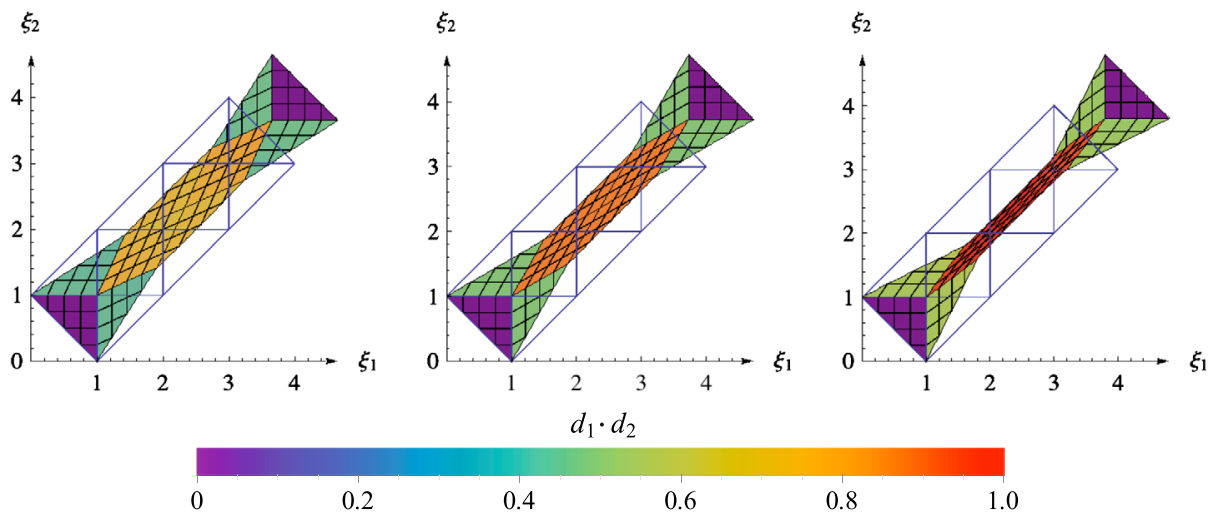

Fig. 7 Deformed shape for imposed displacements $u_{0}$ of: $0.65,0.73$ and 0.8
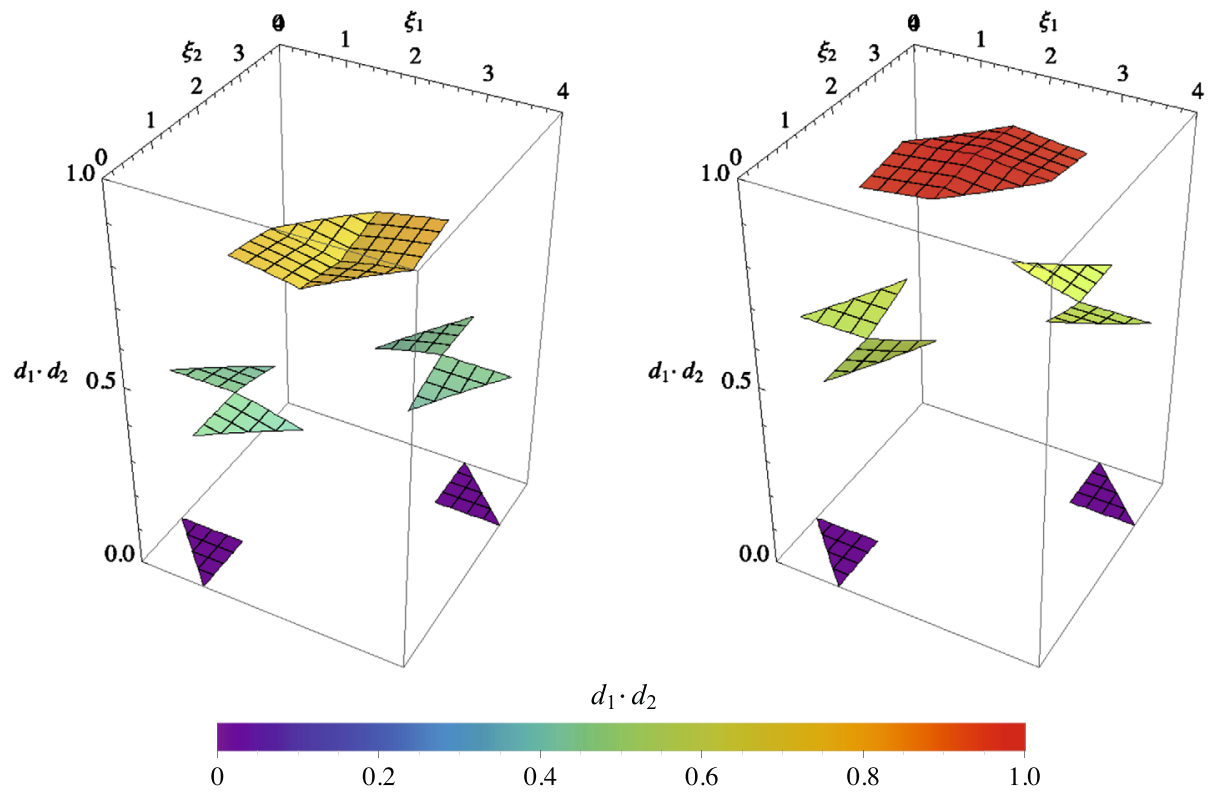

Fig. 8 Cosine of the angle between the fibers for an imposed displacement $u_{0}$ of: 0.65 and 0.8

This numerical results raises questions about its causes and implications. The questions to be addressed are, in particular, the following ones: Can the detected numerical behavior be related to some physical phenomena? Are these phenomena present in the fiber reinforcements which originated our investigations? Which complex mechanical structures show the behavior detected by the model which we consider in the present paper?

The values of $\mathbf{d}_{1} \cdot \mathbf{d}_{2}$ are plotted for the entire reference specimen in Figs. 8 and 9, and, for particular sections, in Fig. 10 to highlight the meaningful entity of the angle variation in each region. It is possible to determine the critical value of displacement which corresponds to the change of concavity. This value is approximately a displacement with dimensionless 
Fig. 9 The critical value 0.73
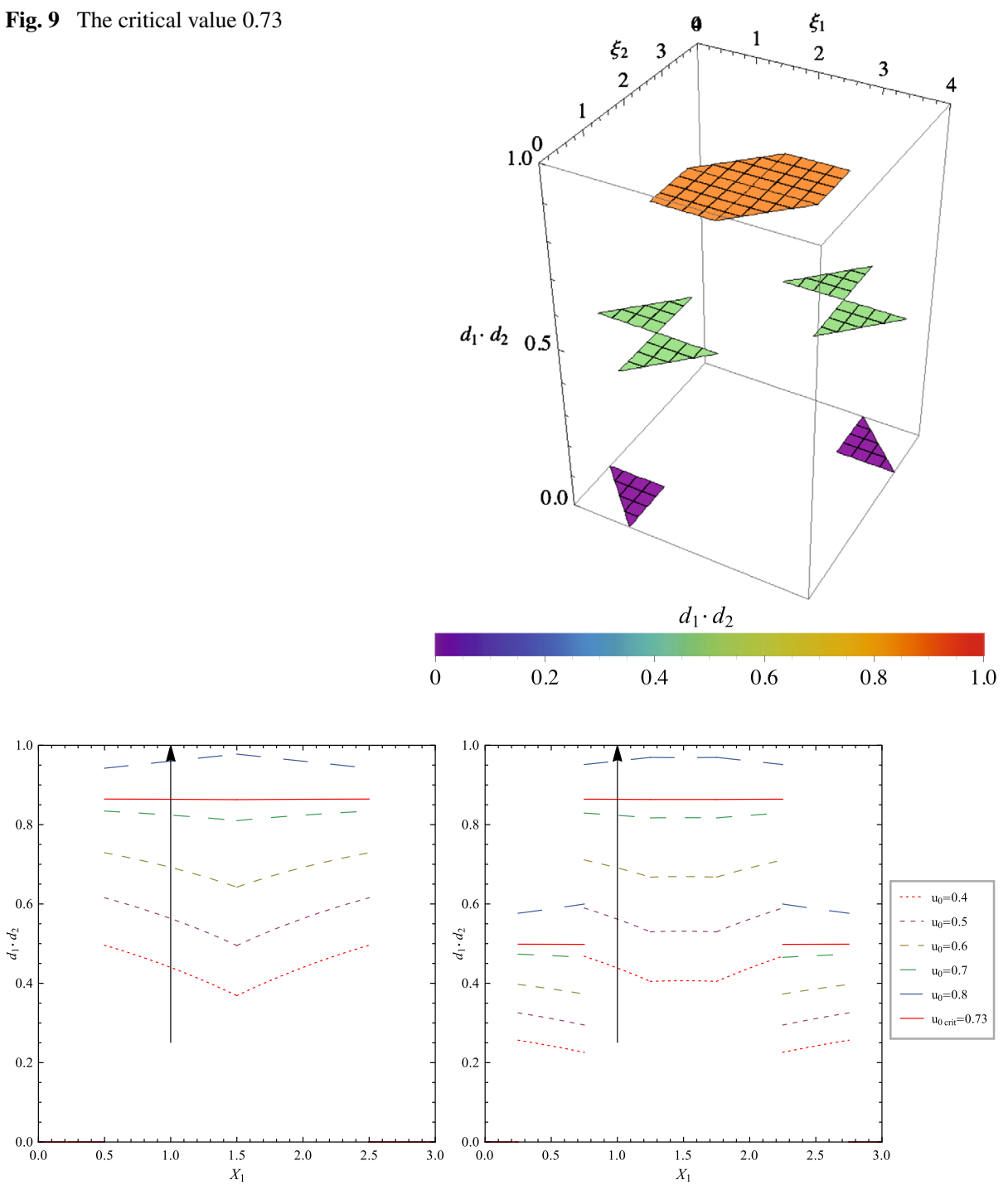

Fig. 10 Cosine of the angle between the fibers in the symmetry axis $X_{1}$ and in the section $X_{2}=0.25$

value of 0.73 (which corresponds about to one quarter of the length of the specimen) and we can see in the presented figures that for the critical displacement $\mathbf{d}_{1} \cdot \mathbf{d}_{2}$ indeed remains constant in all the different zones of the specimen.

\section{Conclusion}

In the present paper the standard bias extension test for first gradient continua with inextensible fibers was studied assuming that the only deformation mechanism related to deformation energy consists in the change of angle between the fibers occurring in the passage from ref- 
erence to current configuration: this change may be interpreted as a shear deformation of the considered continuum.

Such a continuum seems suitable to describe the behavior of some complex materials in which very stiff fibers are embedded and can be applied, with some cautions, to describe the mechanical behavior of some fiber reinforcements. The reader should recall that this study was limited to the analysis of planar deformations of a 2D plane continuum. Actually, in the case of out-of-plane motions, the Rivlin representation for placement fields does not hold and the considered system shows a much more complex behavior and will surely show bifurcations and instabilities also due to small losses of symmetry: post-buckling analysis will then become unavoidable (for the study of some systems showing similar static and dynamic instability phenomena, also in the presence of inextensibility constraints, the reader is referred, e.g., to [43-45, 48, 49]).

The interest of results presented here could go far beyond the context considered up to now: (i) electrospinning (see, e.g., [50]) opens unexpected possibilities to the constructions of fabrics whose lower length-scale could go up to the nanometers (the most recent formulations of the theory of nearly-inextensible nano-fibers and nano-beams can be found in $[12,24,25,75]$; (ii) the most recent investigations have shown that many biological tissues incorporate nearly-inextensible fibers and consequently many models have been presented in different papers to account for their presence [28-30, 33, 34, 76]. Therefore, the insight gained in the present study may produce some impact in other fields of application: (i) biological systems have been optimized by natural selection to resist externally applied loads with a minimum expense of material; hence they have the same mechanical properties as composite reinforcements, (ii) reinforcements fabrics are more and more often woven at nanoscale; while the inextensibility constraint still plays a relevant role, it is not clear what are the limits of applicability of classical mechanics in this case (see, e.g., [12]), (iii) the microstructure of fabrics does allow for instability phenomena at microlevel which may initiate related instabilities at macro-level.

The kinematics considered in this paper is rather restrictive, even if the assumption of exact inextensibility of orthogonal families of fibers seems to be rather wellgrounded from a physical point of view. Also the deformation energy considered (which depends quadratically only on the variation of the shear angle between the inextensible fibers) does not seem sufficient to describe completely many experimental observations $[9,11,14,31,35,36,41,42]$. In a future investigation, we plan to study a continua with extensible or inextensible fibers whose energy depend also on higher displacement gradients (by using the methods presented, e.g., in [20, 21, 23], or in [37, 38, 54, 56, 65]).

The simple first gradient continuum considered here has been introduced mainly to move a first step towards an effective modeling of the bias extension tests for fiber reinforcements or for newly conceived and built metamaterials. However the work here seems rich enough to catch some peculiarities of the mechanical behavior of fiber reinforcements. Indeed our results predict a sharp discontinuity in the angle variation (shear deformation between inextensible fibers) in the passage between the different (as kinematically characterized in terms of the imposed boundary conditions) zones of the specimen undergoing the bias test. This circumstance catches some features of the experimental observation: indeed, in [31] the extension bias test shows shear deformation gradients that are concentrated in narrow regions in the neighborhood of some inextensible fibers. On the other hand the thickness of these regions is experimentally seen to be non-vanishing: therefore it seems a well-grounded conjecture to state that at least second gradient energies $[19,69]$ are needed if one wants to get models capable to predict the onset of the boundary layers where high gradients of shear angle are concentrated. 
Here it has to be underlined, once more, that it is very difficult to characterize, for considered mechanical systems, equilibrium configurations as those configurations where balance of force and momentum is to be satisfied. Indeed, as already implied in [72, 78], the postulation of continuum mechanics based on balance equations does not seem adapted to formulate the models needed here: the difficulties in formulating balance laws when introducing Lagrange multipliers in order to account for all possible contact actions can be insurmountable. Only when mechanics is based on suitable variational principles then well-posed mathematical problems are easily obtained: the continuum models formulated in [1, 26, 52, 54-56, 59$61,63,66-68,70,71]$ give simply a further example of this statement.

In future papers we will aim to analyze the cases of:

- a pure second gradient energy model for the standard bias extension test that describes the onset of boundary layers that characterize the experimental behavior (the basic results to formulate these models are presented, e.g., in $[6,18,80]$ );

- a mixed first-second (or possibly even higher) gradient energy model for the standard bias extension test (as proposed, e.g., in [20-23]);

- a finite element scheme to study the bias extension test and its comparison with the model hereby presented following the methods presented, e.g., in $[8,13,15,32]$ : the reader should note that the Euler-Lagrange equations presented here are strongly non-linear and definitively non-local. As a consequence, even when a local model can be formulated in alternative, it will produce extremely stiff numerical problems. The development of suitable ad hoc integration schemes will be needed: their capability of handling loss of regularity should be useful in the presented context;

- a bias extension test with an asymmetric imposed displacement in which anisotropies will play even a greater role (see, e.g., [5] for the development of some of the required conceptual tools);

- a model for the bias extension test allowing for a 3D deformation and therefore capable to model an eventual out of plane wrinkling [37-40, 58, 62, 73, 77, 78] and the related buckling problems (to be studied, e.g., by means of the methods presented in [43, 45]);

- a bias extension test in which the length ratio of the specimen is an arbitrary number greater than to 2 ;

- a 3D composite interlock subjected to a 3 point bending test to be compared with the experimental results $[14,46]$;

- the "stamping" construction process or the study of fabrics in which the fibers are interacting because of contact interactions involving local deformation and friction: this can be done by taking into consideration the models and the methods which are presented in $[2-4,10]$.

Acknowledgements The authors must thank the Università di Roma La Sapienza and the MEMOCS Research Centre for their support. The fruitful scientific discussions with P. Seppecher, C. Boutin, A. Luongo, U. Andreaus, M. Cuomo, A. Cazzani, L. Lozzi and E. Turco greatly influenced the investigations which were presented in this paper.

\section{Appendix}

In this appendix we supply some more details about the derivation of the Euler-Lagrange conditions used in the paper. 


\subsection{Deformation Energy Functional}

The functional whose first variation has to be calculated is:

$$
\begin{aligned}
\mathscr{A}_{W}(\mu(\cdot))= & \int_{B} W d m+\Lambda C\left(\mu_{, \xi}\right) \\
= & \int_{B}\left[\frac { 1 } { 2 } \left(\left(\mu_{, \xi}\left(\xi_{1}\right)\right)^{2}\left(1-\left(\mu_{, \xi}\left(\xi_{2}\right)\right)^{2}\right)+\left(\mu_{, \xi}\left(\xi_{2}\right)\right)^{2}\left(1-\left(\mu_{, \xi}\left(\xi_{1}\right)\right)^{2}\right)\right.\right. \\
& \left.+2 \mu_{, \xi}\left(\xi_{1}\right) \mu_{, \xi}\left(\xi_{2}\right) \sqrt{\left.\left(1-\left(\mu_{, \xi}\left(\xi_{1}\right)\right)^{2}\right)\left(1-\left(\mu_{, \xi}\left(\xi_{2}\right)\right)^{2}\right)\right)}\right] d m \\
& +\left(\mu(H-1)+\int_{1}^{3} \sqrt{1-\left(\mu_{, \xi}\right)^{2}} d \eta-3-u_{0}\right) \Lambda .
\end{aligned}
$$

Remark The reader should note that this functional maps a real function $\mu$ defined in the interval $[1,3]$ into a real number. However the function $\mu$ is calculated in some occurrences in the variable $\xi_{1}$ and in some other occurrences in the variable $\xi_{2}$ when forming the integrand function appearing inside the square brackets of the previous equation. This integrand is indeed a function of the two variables $\left(\xi_{1}, \xi_{2}\right)$ and its domain of integration is the twodimensional domain $B$.

Considering the partition (17) of the domain B and defining $\underline{\Delta}=\Delta_{11} \cup \Delta_{21} \cup \Delta_{22} \cup \Delta_{21}$, it is possible to rewrite the functional as follows:

$$
\begin{aligned}
\mathscr{A}_{W}= & \int_{\Delta_{01} \cup \Delta_{32}} \frac{1}{2}\left(1-\left(\mu_{, \xi}\left(\xi_{2}\right)\right)^{2}\right) d m+\int_{\Delta_{10} \cup \Delta_{23}} \frac{1}{2}\left(1-\left(\mu_{, \xi}\left(\xi_{1}\right)\right)^{2}\right) d m \\
& +\int_{\underline{\Delta}} \frac{1}{2}\left[\left(\mu_{, \xi}\left(\xi_{1}\right)\right)^{2}\left(1-\left(\mu_{, \xi}\left(\xi_{2}\right)\right)^{2}\right)+\left(\mu_{, \xi}\left(\xi_{2}\right)\right)^{2}\left(1-\left(\mu_{, \xi}\left(\xi_{1}\right)\right)^{2}\right)\right. \\
& \left.+2 \mu_{, \xi}\left(\xi_{1}\right) \mu_{, \xi}\left(\xi_{2}\right) \sqrt{\left(1-\left(\mu_{, \xi}\left(\xi_{1}\right)\right)^{2}\right)\left(1-\left(\mu_{, \xi}\left(\xi_{2}\right)\right)^{2}\right)}\right] d m \\
& +\left(\int_{1}^{3}\left(\mu_{, \xi}\left(\xi_{1}\right)+\sqrt{1-\left(\mu_{, \xi}\left(\xi_{1}\right)\right)^{2}}\right) d \xi_{1}-2-u_{0}\right) \Lambda,
\end{aligned}
$$

which, by using the assumed symmetry of the solution, becomes

$$
\begin{aligned}
\mathscr{A}_{W}= & \int_{\Delta_{01} \cup \Delta_{32}} \frac{1}{2}\left(1-\left(\mu_{, \xi}\left(\xi_{2}\right)\right)^{2}\right) d m+\int_{\Delta_{10} \cup \Delta_{23}} \frac{1}{2}\left(1-\left(\mu_{, \xi}\left(\xi_{1}\right)\right)^{2}\right) d m \\
& +\int_{\underline{\Delta}}\left[\left(\mu_{, \xi}\left(\xi_{1}\right)\right)^{2}\left(1-\left(\mu_{, \xi}\left(\xi_{2}\right)\right)^{2}\right)+\mu_{, \xi}\left(\xi_{1}\right) \mu_{, \xi}\left(\xi_{2}\right)\right. \\
& \left.\times \sqrt{\left(1-\left(\mu_{, \xi}\left(\xi_{1}\right)\right)^{2}\right)\left(1-\left(\mu_{, \xi}\left(\xi_{2}\right)\right)^{2}\right)}\right] d m \\
& +\left(\int_{1}^{3}\left(\mu_{, \xi}\left(\xi_{1}\right)+\sqrt{1-\left(\mu_{, \xi}\left(\xi_{1}\right)\right)^{2}}\right) d \xi_{1}-2-u_{0}\right) \Lambda .
\end{aligned}
$$


6.2 Calculation of the First Variation of the Deformation Energy Functional

The first variation of the deformation energy functional can be expressed as:

$$
\delta \mathscr{A}_{W}=\int_{B} \delta W d m+\Lambda \delta C\left(\mu_{, \xi}\right)+C(\mu, \xi) \delta \Lambda .
$$

We have

$$
\begin{array}{rl}
\int_{B} \delta W & d m+\Lambda \delta C\left(\mu_{, \xi}\right) \\
= & -\int_{\Delta_{01} \cup \Delta_{32}} \mu_{, \xi}\left(\xi_{2}\right) \delta \mu_{, \xi}\left(\xi_{2}\right) d m-\int_{\Delta_{10} \cup \Delta_{23}} \mu_{, \xi}\left(\xi_{1}\right) \delta \mu_{, \xi}\left(\xi_{1}\right) d m \\
& +\int_{\underline{\Delta}}\left(2 \mu_{, \xi}\left(\xi_{1}\right)\left(1-\left(\mu_{, \xi}\left(\xi_{2}\right)\right)^{2}\right)+\frac{1-2\left(\mu_{, \xi}\left(\xi_{1}\right)\right)^{2}}{\sqrt{1-\left(\mu_{, \xi}\left(\xi_{1}\right)\right)^{2}}} \mu_{, \xi}\left(\xi_{2}\right) \sqrt{1-\left(\mu_{, \xi}\left(\xi_{2}\right)\right)^{2}}\right. \\
& \left.+\left(1-\frac{\mu_{, \xi}\left(\xi_{1}\right)}{\sqrt{1-\left(\mu_{, \xi}\left(\xi_{1}\right)\right)^{2}}}\right) \Lambda\right) \delta \mu_{, \xi}\left(\xi_{1}\right) \\
& +\int_{\underline{\Delta}}\left(-2 \mu_{, \xi}\left(\xi_{2}\right)\left(\left(\mu_{, \xi}\left(\xi_{1}\right)\right)^{2}\right)+\frac{1-2\left(\mu_{, \xi}\left(\xi_{2}\right)\right)^{2}}{\sqrt{1-\left(\mu_{, \xi}\left(\xi_{2}\right)\right)^{2}}} \mu_{, \xi}\left(\xi_{1}\right)\right. \\
& \left.\times \sqrt{1-\left(\mu_{, \xi}\left(\xi_{1}\right)\right)^{2}}\right) \delta \mu_{, \xi}\left(\xi_{2}\right) d m
\end{array}
$$

Due to the symmetry condition, we have that

$$
\begin{aligned}
& \int_{\underline{\Delta}}\left(-2 \mu_{, \xi}\left(\xi_{2}\right)\left(\left(\mu_{, \xi}\left(\xi_{1}\right)\right)^{2}\right)+\frac{1-2\left(\mu_{, \xi}\left(\xi_{2}\right)\right)^{2}}{\sqrt{1-\left(\mu_{, \xi}\left(\xi_{2}\right)\right)^{2}}} \mu_{, \xi}\left(\xi_{1}\right) \sqrt{1-\left(\mu_{, \xi}\left(\xi_{1}\right)\right)^{2}}\right) \delta \mu_{, \xi}\left(\xi_{2}\right) d m \\
& =\int_{\underline{\Delta}}\left(-2 \mu_{, \xi}\left(\xi_{1}\right)\left(\left(\mu_{, \xi}\left(\xi_{2}\right)\right)^{2}\right)+\frac{1-2\left(\mu_{, \xi}\left(\xi_{1}\right)\right)^{2}}{\sqrt{1-\left(\mu_{, \xi}\left(\xi_{1}\right)\right)^{2}}} \mu_{, \xi}\left(\xi_{2}\right) \sqrt{1-\left(\mu_{, \xi}\left(\xi_{2}\right)\right)^{2}}\right) \delta \mu_{, \xi}\left(\xi_{1}\right) d m,
\end{aligned}
$$

and

$$
\int_{\Delta_{01} \cup \Delta_{32}} \mu_{, \xi}\left(\xi_{2}\right) \delta \mu_{, \xi}\left(\xi_{2}\right) d m=\int_{\Delta_{10} \cup \Delta_{23}} \mu_{, \xi}\left(\xi_{1}\right) \delta \mu_{, \xi}\left(\xi_{1}\right) d m .
$$

So, replacing (62) and (63) in (61), we obtain:

$$
\begin{array}{rl}
\int_{B} \delta W & d m+\Lambda \delta C\left(\mu_{, \xi}\right) \\
= & -2 \int_{\Delta_{10} \cup \Delta_{23}} \mu_{, \xi}\left(\xi_{1}\right) \delta \mu_{, \xi}\left(\xi_{1}\right) d m+\int_{\underline{\Delta}}\left(2 \mu_{, \xi}\left(\xi_{1}\right)-4 \mu_{, \xi}\left(\xi_{1}\right)\left(\mu_{, \xi}\left(\xi_{2}\right)\right)^{2}\right. \\
& \left.+2 \frac{1-2\left(\mu_{, \xi}\left(\xi_{1}\right)\right)^{2}}{\sqrt{1-\left(\mu_{, \xi}\left(\xi_{1}\right)\right)^{2}}} \mu_{, \xi}\left(\xi_{2}\right) \sqrt{1-\left(\mu_{, \xi}\left(\xi_{2}\right)\right)^{2}}+\left(1-\frac{\mu_{, \xi}\left(\xi_{1}\right)}{\sqrt{1-\left(\mu_{, \xi}\left(\xi_{1}\right)\right)^{2}}}\right) \Lambda\right) \delta \mu_{, \xi}\left(\xi_{1}\right) .
\end{array}
$$


Now, applying the Fubini Theorem we find:

$$
\begin{aligned}
\int_{B} \delta W d m & +\Lambda \delta C\left(\mu_{, \xi}\right) \\
= & \int_{1}^{2}\left[4 \mu\left(\xi_{1}\right)\left(-1+\int_{1}^{1+\xi_{1}}\left(1-\left(\mu_{, \xi}\left(\xi_{2}\right)\right)^{2}\right) d \xi_{2}\right)\right. \\
& +2 \frac{1-2\left(\mu_{, \xi}\left(\xi_{1}\right)\right)^{2}}{\sqrt{1-\left(\mu_{, \xi}\left(\xi_{1}\right)\right)^{2}}} \int_{1}^{1+\xi_{1}} \mu_{, \xi}\left(\xi_{2}\right) \sqrt{1-\left(\mu_{, \xi}\left(\xi_{2}\right)\right)^{2}} d \xi_{2} \\
& \left.+\left(1-\frac{\mu_{, \xi}\left(\xi_{1}\right)}{\sqrt{1-\left(\mu_{, \xi}\left(\xi_{1}\right)\right)^{2}}}\right) \Lambda\right] \delta \mu_{, \xi}\left(\xi_{1}\right) d \xi_{1} \\
& +\int_{2}^{3}\left[4 \mu_{, \xi}\left(\xi_{1}\right)\left(-1+\int_{\xi_{1}-1}^{3}\left(1-\left(\mu_{, \xi}\left(\xi_{2}\right)\right)^{2}\right) d \xi_{2}\right)\right. \\
& +2 \frac{1-2\left(\mu_{, \xi}\left(\xi_{1}\right)\right)^{2}}{\sqrt{1-\left(\mu_{, \xi}\left(\xi_{1}\right)\right)^{2}}} \int_{\xi_{1}-1}^{3} \mu_{, \xi}\left(\xi_{2}\right) \sqrt{1-\left(\mu_{, \xi}\left(\xi_{2}\right)\right)^{2}} d \xi_{2} \\
& \left.+\left(1-\frac{\mu_{, \xi}\left(\xi_{1}\right)}{\sqrt{1-\left(\mu_{, \xi}\left(\xi_{1}\right)\right)^{2}}}\right) \Lambda\right] \delta \mu_{, \xi}\left(\xi_{1}\right) d \xi_{1} .
\end{aligned}
$$

Renaming the variables by means of the equalities $\xi_{2}=\eta$ and $\xi_{1}=\xi$, we are lead to define two functions $F_{i}$ in the following way:

$$
\begin{aligned}
F_{1}\left(\mu_{, \xi}, \xi, \Lambda\right)= & \frac{1-2\left(\mu_{, \xi}(\xi)\right)^{2}}{2 \sqrt{1-\left(\mu_{, \xi}(\xi)\right)^{2}}} \underbrace{\int_{1}^{1+\xi} \mu_{, \xi}(\eta) \sqrt{1-\left(\mu_{, \xi}(\eta)\right)^{2}} d \eta}_{a_{1}(\xi)}-\mu(\xi) \\
& \times \underbrace{\left(1-\int_{1}^{1+\xi}\left(1-\left(\mu_{, \xi}(\eta)\right)^{2}\right) d \eta\right)}_{d_{1}(\xi)}+\left(1-\frac{\mu_{, \xi}(\xi)}{\sqrt{1-\left(\mu_{, \xi}(\xi)\right)^{2}}}\right) \Lambda, \\
F_{2}\left(\mu_{, \xi}, \xi, \Lambda\right)= & \frac{1-2\left(\mu_{, \xi}(\xi)\right)^{2}}{2 \sqrt{1-\left(\mu_{, \xi}(\xi)\right)^{2}}} \underbrace{\int_{\xi-1}^{3} \mu_{, \xi}(\eta) \sqrt{1-\left(\mu_{, \xi}(\eta)^{2}\right.} d \eta-\mu_{, \xi}(\xi)}_{a_{2}(\xi)} \\
& \times \underbrace{\left(1-\int_{\xi-1}^{3}\left(1-\left(\mu_{, \xi}(\eta)\right)^{2}\right) d \eta\right)}_{d_{2}(\xi)}+\left(1-\frac{\mu_{, \xi}(\xi)}{\sqrt{1-\left(\mu_{, \xi}(\xi)\right)^{2}}}\right) \Lambda .
\end{aligned}
$$

Therefore the first variation of the deformation energy can be represented as follows:

$$
\delta \mathscr{A}_{W}=4 \int_{1}^{2} F_{1}(\mu, \xi, \xi, \Lambda) \delta \mu_{, \xi} d \xi+4 \int_{2}^{3} F_{2}\left(\mu_{, \xi}, \xi, \Lambda\right) \delta \mu_{, \xi} d \xi+C\left(\mu_{, \xi}\right) \delta \Lambda
$$


Applying an integration by parts, we find that it is possible to rewrite the variation of the deformation energy functional as follows:

$$
\begin{aligned}
\delta \mathscr{A}_{W}= & 4 \int_{1}^{2} F_{1}\left(\mu_{, \xi}, \xi, \Lambda\right) \delta \mu_{, \xi} d \xi+4 \int_{2}^{3} F_{2}(\mu, \xi, \xi, \Lambda) \delta \mu_{, \xi} d \xi+C(\mu, \xi) \delta \Lambda \\
= & 4\left[F_{1}\left(\mu_{, \xi}, \xi, \Lambda\right) \delta \mu\right]_{1}^{2}+4\left[F_{2}\left(\mu_{, \xi}, \xi, \Lambda\right) \delta \mu\right]_{2}^{3}-4 \int_{1}^{2} \frac{\partial F_{1}(\mu, \xi, \xi, \Lambda)}{\partial \xi} \delta \mu d \xi \\
& -4 \int_{2}^{3} \frac{\partial F_{2}\left(\mu_{, \xi}, \xi, \Lambda\right)}{\partial \xi} \delta \mu d \xi+C\left(\mu_{, \xi}\right) \delta \Lambda .
\end{aligned}
$$

Because of the arbitrariness of the variation $\delta \mu$, we get that:

$$
\left\{\begin{array}{lll}
\frac{\partial F_{i}(\mu, \xi, \xi, \Lambda)}{\partial \xi}=0 & \forall \xi \in I_{i} \quad \Longrightarrow \quad F_{i}\left(\mu_{, \xi}, \xi, \Lambda\right)=K_{i} \\
F_{2}(\mu, \xi, 3, \Lambda)=0 & \Longrightarrow & K_{2}=0 \\
F_{1}(\mu, \xi, 2, \Lambda)=F_{2}(\mu, \xi, 2, \Lambda) & \Longrightarrow & K_{1}=K_{2}=0 \\
C(\mu, \xi)=0 . & &
\end{array}\right.
$$

Therefore, the stationarity condition is given by the system of equations:

$$
\left\{\begin{array}{l}
F_{i}\left(\mu_{, \xi}, \xi, \Lambda\right)=0 \quad \forall \xi \in I_{i} \\
C\left(\mu_{, \xi}\right)=0
\end{array}\right.
$$

with $I_{1}=[1,2], I_{2}=[2,3]$.

\section{References}

1. Adkins, J.E., Rivlin, R.S.: Large elastic deformations of isotropic materials X. Reinforcement by inextensible cords. Philos. Trans. R. Soc. Lond. Ser. A, Math. Phys. Sci. 248(944), 201-223 (1955)

2. Andreaus, U., Chiaia, B., Placidi, L.: Soft-impact dynamics of deformable bodies. Contin. Mech. Thermodyn. 25(2-4), 375-398 (2013)

3. Andreaus, U., Giorgio, I., Lekszycki, T.: A 2-D continuum model of a mixture of bone tissue and bioresorbable material for simulating mass density redistribution under load slowly variable in time. Z. Angew. Math. Mech. 94(12), 978-1000 (2014)

4. Andreaus, U., Placidi, L., Rega, G.: Numerical simulation of the soft contact dynamics of an impacting bilinear oscillator. Commun. Nonlinear Sci. Numer. Simul. 15(9), 2603-2616 (2010)

5. Auffray, N.: On the algebraic structure of isotropic generalized elasticity theories. Math. Mech. Solids (2013). doi:10.1177/1081286513507941

6. Auffray, N., dell'Isola, F., Eremeyev, V.A., Madeo, A., Rosi, G.: Analytical continuum mechanics à la Hamilton-Piola least action principle for second gradient continua and capillary fluids. Math. Mech. Solids 20(4), 375-417 (2015)

7. Ball, J.M.: Convexity conditions and existence theorems in nonlinear elasticity. Arch. Ration. Mech. Anal. 63, 337-403 (1977)

8. Baraldi, D., Reccia, E., Cazzani, A., Cecchi, A.: Comparative analysis of numerical discrete and finite element models: The case of in-plane loaded periodic brickwork. Composites: Mechanics, Computations, Applications, An International Journal 4(4), 319-344 (2013)

9. Bel, S., Boisse, P., Dumont, F.: Analyses of the deformation mechanisms of non-crimp fabric composite reinforcements during preforming. Appl. Compos. Mater. 19(3-4), 513-528 (2012) 
10. Bersani, A.M., Giorgio, I., Tomassetti, G.: Buckling of an elastic hemispherical shell with an obstacle. Contin. Mech. Thermodyn. 25(2-4), 443-467 (2013)

11. Cao, J., Akkerman, R., Boisse, P., Chen, J., Cheng, H.S., de Graaf, E.F., Gorczyca, J.L., Harrison, P., Hivet, G., Launay, J., Lee, W., Liu, L., Lomov, S.V., Long, A., de Luycker, E., Morestin, F., Padvoiskis, J., Peng, X.Q., Sherwood, J., Stoilova, Tz., Tao, X.M., Verpoest, I., Willems, A., Wiggers, J., Yu, T.X., Zhu, B.: Characterization of mechanical behavior of woven fabrics: experimental methods and benchmark results. Composites, Part A, Appl. Sci. Manuf. 39(6), 1037-1053 (2008)

12. Carcaterra, A.: Quantum Euler beam-QUEB: modeling nanobeams vibration. Contin. Mech. Thermodyn. 27(1-2), 145-156 (2015)

13. Cazzani, A., Malagù, M., Turco, E.: Isogeometric analysis of plane-curved beams. Math. Mech. Solids (2014). doi:10.1177/1081286514531265

14. Charmetant, A., Boisse, P., Orliac, J.G., Vidal Sallée, E.: Hyperelastic model for large deformation analyses of 3D interlock composite preforms. Compos. Sci. Technol. 72, 1352-1360 (2012)

15. Cuomo, M., Contrafatto, L., Greco, L.: A variational model based on isogeometric interpolation for the analysis of cracked bodies. Int. J. Eng. Sci. 80, 173-188 (2014)

16. d'Agostino, M.V., Giorgio, I., Greco, L., Madeo, A., Boisse, P.: Continuum and discrete models for structures including (quasi-)inextensible elasticae with a view to the design and modeling of composite reinforcements. Int. J. Solids Struct. 59, 1-17 (2015)

17. Davini, C., Governatori, P.: Nets with hexagonal cell structure. J. Elast. 92(1), 35-59 (2008)

18. dell'Isola, F., Sciarra, G., Vidoli, S.: Generalized Hooke's law for isotropic second gradient materials. Proc. R. Soc., Math. Phys. Eng. Sci. 465(2107), 2177-2196 (2009)

19. dell'Isola, F., Gouin, H., Rotoli, G.: Nucleation of spherical shell-like interfaces by second gradient theory: numerical simulations. Eur. J. Mech. B, Fluids 15(4), 545-568 (1996)

20. dell'Isola, F., Seppecher, P.: The relationship between edge contact forces, double forces and interstitial working allowed by the principle of virtual power. C. R. Acad. Sci., Sér. IIb Méc. Phys. Astron. (1995)

21. dell'Isola, F., Seppecher, P.: Edge contact forces and quasi-balanced power. Meccanica 32(1), 33-52 (1997)

22. dell'Isola, F., Seppecher, P., Madeo, A.: How contact interactions may depend on the shape of Cauchy cuts in Nth gradient continua: approach “à la D'Alembert”. Z. Angew. Math. Phys. 63(6), 1119-1141 (2012)

23. dell'Isola, F., Steigmann, D.: A two-dimensional gradient-elasticity theory for woven fabrics. J. Elast. 118(1), 113-125 (2015)

24. Eremeyev, V.A., Ivanova, E.A., Morozov, N.F., Strochkov, S.E.: Natural vibrations of nanotubes. Dokl. Phys. 52(8), 431-435 (2007)

25. Eremeyev, V.A., Ivanova, E.A., Morozov, N.F., Strochkov, S.E.: Natural vibrations in a system of nanotubes. J. Appl. Mech. Tech. Phys. 49(2), 291-300 (2008)

26. Ericksen, J.L., Rivlin, R.S.: Large elastic deformations of homogeneous anisotropic materials. J. Ration. Mech. Anal. 3(3), 281-301 (1954)

27. Everstine, G.C., Pipkin, A.C.: Boundary layers in fiber-reinforced materials. J. Appl. Mech. 40(2), 518$522(1973)$

28. Federico, S., Herzog, W.: On the anisotropy and inhomogeneity of permeability in articular cartilage. Biomech. Model. Mechanobiol. 7, 367-378 (2008)

29. Federico, S., Herzog, W.: On the permeability of fibre-reinforced porous materials. Int. J. Solids Struct. 45, 2160-2172 (2008)

30. Federico, S., Grillo, A.: Elasticity and permeability of porous fibre-reinforced materials under large deformations. Mech. Mater. 44, 58-71 (2012)

31. Ferretti, M., Madeo, A., dell'Isola, F., Boisse, P.: Modeling the onset of shear boundary layers in fibrous composite reinforcements by second-gradient theory. Z. Angew. Math. Phys. 65(3), 587-612 (2014)

32. Greco, L., Cuomo, M.: An implicit G1 multi patch B-spline interpolation for Kirchhoff-Love space rod. Comput. Methods Appl. Mech. Eng. 269, 173-197 (2014)

33. Grillo, A., Federico, S., Wittum, G.: Growth, mass transfer, and remodeling in fiber-reinforced, multiconstituent materials. Int. J. Non-Linear Mech. 47, 388-401 (2012)

34. Grillo, A., Federico, S., Wittum, G.: Growth, mass transfer, and remodeling in fiber-reinforced, multiconstituent materials. Int. J. Non-Linear Mech. 47(2), 388-401 (2012)

35. Hamila, N., Boisse, P.: Locking in simulation of composite reinforcement deformations. analysis and treatment. Composites, Part A, Appl. Sci. Manuf. 53, 109-117 (2013)

36. Hamila, N., Boisse, P.: Tension locking in finite-element analyses of textile composite reinforcement deformation. C. R., Méc. 341(6), 508-519 (2013)

37. Hilgers, M.G., Pipkin, A.C.: Elastic sheets with bending stiffness. Q. J. Mech. Appl. Math. 45(1), 57-75 (1992) 
38. Hilgers, M.G., Pipkin, A.C.: Energy-minimizing deformations of elastic sheets with bending stiffness. J. Elast. 31(2), 125-139 (1993)

39. Hilgers, M.G., Pipkin, A.C.: Bending energy of highly elastic membranes II. Q. Appl. Math. 54(2), 307-316 (1996)

40. Hu, M.Z., Kolsky, H., Pipkin, A.C.: Bending theory for fiber-reinforced beams. J. Compos. Mater. 19(3), 235-249 (1985)

41. Launay, J., Hivet, G., Duong, A.V., Boisse, P.: Experimental analysis of the influence of tensions on in plane shear behavior of woven composite reinforcements. Compos. Sci. Technol. 68(2), 506-515 (2008)

42. Lee, W., Padvoiskis, J., Cao, J., de Luycker, E., Boisse, P., Morestin, F., Chen, J., Sherwood, J.: Biasextension of woven composite fabrics. Int. J. Material Form. 1(1), 895-898 (2008)

43. Luongo, A.: Eigensolutions of perturbed nearly defective matrices. J. Sound Vib. 185(3), 377-395 (1995)

44. Luongo, A., Paolone, A., Piccardo, G.: Postcritical behavior of cables undergoing two simultaneous galloping modes. Meccanica 33(3), 229-242 (1998)

45. Luongo, A., Plgnataro, M.: On the perturbation analysis of interactive buckling in nearly symmetric structures. Int. J. Solids Struct. 29(6), 721-733 (1992)

46. Madeo, A., Ferretti, M., dell'Isola, F., Boisse, P.: Thick fibrous composite reinforcements behave as special second gradient materials: three point bending of 3D interlocks. Z. Angew. Math. Phys. (2015). doi: 10.1007/s00033-015-0496-z

47. Paroni, R.: An existence theorem for inextensible nets with slack. Math. Mech. Solids 17(5), 460-472 (2012)

48. Piccardo, G., Ranzi, G., Luongo, A.: A complete dynamic approach to the generalized beam theory cross-section analysis including extension and shear modes. Math. Mech. Solids (2013). doi: $10.1177 / 1081286513493107$

49. Piccardo, G., Ranzi, G., Luongo, A.: A direct approach for the evaluation of the conventional modes within the gbt formulation. Thin-Walled Struct. 74, 133-145 (2014)

50. Piperno, S., Lozzi, L., Rastelli, R., Passacantando, M., Santucci, S.: PMMA nanofibers production by electrospinning. Appl. Surf. Sci. 252(15), 5583-5586 (2006)

51. Pipkin, A.C.: Finite deformations of ideal fiber-reinforced composites. In: Mechanics of Composite Materials, pp. 251-308. Academic Press, Inc., New York (1974)

52. Pipkin, A.C.: Generalized plane deformations of ideal fiber-reinforced materials. Q. Appl. Math. 32, 253-263 (1974)

53. Pipkin, A.C.: Finite deformations in materials reinforced with inextensible cords. Finite Elast. 27, 92$102(1977)$

54. Pipkin, A.C.: Energy changes in ideal fiber-reinforced composites. Q. Appl. Math. 35, 455-463 (1978)

55. Pipkin, A.C.: Some developments in the theory of inextensible networks. Q. Appl. Math. 38(3), 343-355 (1980)

56. Pipkin, A.C.: Plane traction problems for inextensible networks. Q. J. Mech. Appl. Math. 34(4), 415-429 (1981)

57. Pipkin, A.C.: Stress channelling and boundary layers in strongly anisotropic solids. In: Continuum Theory of the Mechanics of Fibre-Reinforced Composites, pp. 123-145. Springer, Berlin (1984)

58. Pipkin, A.C.: Continuously distributed wrinkles in fabrics. Arch. Ration. Mech. Anal. 95(2), 93-115 (1986)

59. Pipkin, A.C., Rivlin, R.S.: Minimum-weight design for pressure vessels reinforced with inextensible fibers. J. Appl. Mech. 30(1), 103-108 (1963)

60. Pipkin, A.C., Rogers, T.G.: A mixed boundary-value problem for fiber-reinforced materials (mixed boundary value problem for fiber reinforced materials, analyzing shear response in multiply admissible kinematic deformations). Q. Appl. Math. 29, 151-155 (1971)

61. Pipkin, A.C., Rogers, T.G.: Plane deformations of incompressible fiber-reinforced materials. J. Appl. Mech. 38(3), 634-640 (1971)

62. Pipkin, A.C., Rogers, T.G.: Infinitesimal plane wrinkling of inextensible networks. J. Elast. 17(1), 35-52 (1987)

63. Pipkin, A.C.: Stress analysis for fiber-reinforced materials. Adv. Appl. Mech. 19, 1-51 (1979)

64. Pipkin, A.C.: Equilibrium of Tchebychev nets. In: The Breadth and Depth of Continuum Mechanics, pp. 287-303. Springer, Berlin (1986)

65. Pipkin, A.C., Sanchez, V.M.: Existence of solutions of plane traction problems for ideal composites. SIAM J. Appl. Math. 26(1), 213-220 (1974)

66. Rivlin, R.S.: Plane strain of a net formed by inextensible cords. J. Ration. Mech. Anal. 4(6), 951-974 (1955)

67. Rivlin, R.S.: Constitutive equation for a fiber-reinforced lamina. In: IUTAM Symposium on Anisotropy, Inhomogeneity and Nonlinearity in Solid Mechanics, pp. 379-384. Springer, Berlin (1995) 
68. Rivlin, R.S.: Networks of inextensible cords. In: Collected Papers of RS Rivlin, pp. 566-579. Springer, Berlin (1997)

69. Rosi, G., Giorgio, I., Eremeyev, V.A.: Propagation of linear compression waves through plane interfacial layers and mass adsorption in second gradient fluids. Z. Angew. Math. Mech. 93(12), 914-927 (2013)

70. Smith, G.F., Rivlin, R.S.: Stress-deformation relations for anisotropic solids. Arch. Ration. Mech. Anal. 1(1), 107-112 (1957)

71. Smith, G.F., Rivlin, R.S.: The strain-energy function for anisotropic elastic materials. In: Collected Papers of RS Rivlin, pp. 541-559. Springer, Berlin (1997)

72. Spencer, A.J.M., et al.: Continuum Theory of the Mechanics of Fibre-Reinforced Composites. CISM Courses and Lectures, vol. 282. Springer, Berlin (1984)

73. Steigmann, D.J., Pipkin, A.C.: Finite deformations of wrinkled membranes. Q. J. Mech. Appl. Math. 42(3), 427-440 (1989)

74. Steigmann, D.J., Pipkin, A.C.: Equilibrium of elastic nets. Philos. Trans. R. Soc., Math. Phys. Eng. Sci. 335(1639), 419-454 (1991)

75. Thiagarajan, G., Deshmukh, K., Wang, Y., Misra, A., Katz, J.L., Spencer, P.: Nano finite element modeling of the mechanical behavior of biocomposites using multi-scale (virtual internal bond) material models. J. Biomed. Mater. Res., Part A 83(2), 332-344 (2007)

76. Tomic, A., Grillo, A., Federico, S.: Poroelastic materials reinforced by statistically oriented fibresnumerical implementation and application to articular cartilage. IMA J. Appl. Math. 79, 1027-1059 (2014)

77. Wang, W.B., Pipkin, A.C.: Inextensible networks with bending stiffness. Q. J. Mech. Appl. Math. 39(3), 343-359 (1986)

78. Wang, W.B., Pipkin, A.C.: Plane deformations of nets with bending stiffness. Acta Mech. 65(1-4), 263279 (1987)

79. Williams, W.O.: Forces in Tchebychev nets. In: Waves and Stability in Continuous Media. Ser. Adv. Math. Appl. Sci, vol. 4, pp. 408-418 (1991)

80. Yang, Y., Misra, A.: Micromechanics based second gradient continuum theory for shear band modeling in cohesive granular materials following damage elasticity. Int. J. Solids Struct. 49(18), 2500-2514 (2012) 